Supporting Information For:

\title{
Water-Mediated Association Provides an Ion Pair Receptor
}

Frank W. Kotch, Vladimir Sidorov, Yiu-Fai Lam, Katherine J. Kayser, Hojun Li, Mark Kaucher and Jeffery T. Davis*

Department of Chemistry and Biochemistry, University of Maryland, College Park, MD 20742

Contents:

1. Synthetic details and schemes for the preparation of $\mathrm{cG} \mathbf{2}, \mathrm{cA} \mathbf{3}$, and $\mathrm{G} 4$

2. Figures

\section{Synthesis}

General Experimental. ${ }^{1} \mathrm{H}$ NMR spectra were recorded on a Bruker DRX-400 or Bruker Avance 400 operating at $400.13 \mathrm{MHz}$, or on a Bruker DRX-500 operating at $500.13 \mathrm{MHz}$. Chemical shifts are reported in ppm relative to the residual protonated solvent peak. The ${ }^{13} \mathrm{C}$ NMR spectra were recorded on a Bruker DRX-400 instrument operating at $100.61 \mathrm{MHz}$ and chemical shift values are reported in ppm relative to the solvent peak. Fast Atom Bombardment (FAB) mass spectra were recorded on a JEOL SX-102A magnetic sector mass spectrometer. Electrospray (ESI) mass spectra were recorded from MeOH solutions on a Finnigan LCQ mass spectrometer by direct infusion. The samples were ionized by nanoelectrospray and detected in the positive ion mode. Circular dichroism spectra were recorded on an Applied Photophysics 180 PiStar instrument at $25^{\circ} \mathrm{C}$ in a cylindrical $0.1 \mathrm{~mm}$ path length quartz cuvette. Chromatography was performed using 60-200 mesh silica purchased from Baker. Thin layer chromatography was performed on Kieselgel 60 F254 and Uniplate ${ }^{\mathrm{TM}}$ Silica Gel GF silica-coated glass plates and visualized by $\mathrm{UV}$ and $\mathrm{I}_{2}$. Dueterated solvents were purchased from Cambridge Isotope Laboratories. All chemicals and solvents were purchased from Sigma, Fluka, Aldrich or Acros.

5,11,17,23-Tetrakis(butoxymethyl)-25,26,27,28-tetrahydroxycalix[4]arene (6). A solution of p-H-calix[4]arene (7.1 g, $16.7 \mathrm{mmol})$, paraformaldehyde (10.52 g, $351 \mathrm{mmol})$, glacial HOAc $(112 \mathrm{~mL})$, conc. $\mathrm{H}_{3} \mathrm{PO}_{4}(119 \mathrm{~mL})$, and conc. $\mathrm{HCl}(126 \mathrm{~mL})$ in dioxane $(500 \mathrm{~mL})$ was stirred at 
$80{ }^{\circ} \mathrm{C}$ for $12 \mathrm{~h}$. The mixture was concentrated to $300 \mathrm{~mL}$, poured into an ice/water mixture (500 $\mathrm{mL})$ and extracted with $\mathrm{CHCl}_{3}(2 \times 70 \mathrm{~mL})$. The combined organic layers were washed with $\mathrm{H}_{2} \mathrm{O}$ $(100 \mathrm{~mL})$, dried over $\mathrm{Na}_{2} \mathrm{SO}_{4}$ and the solvent was evaporated under reduced pressure to give crude $p$-chloromethyl-calix[4]arene 5 (10.64 g) as a light brown solid. Na (21.3 g, $926 \mathrm{mmol})$ was washed with hexanes (to remove kerosene) and dissolved in $\mathrm{BuOH}(500 \mathrm{~mL})$ to generate $\mathrm{NaOBu}$ in situ. A suspension of crude calixarene 5 (10.64g) in $\mathrm{BuOH}(100 \mathrm{~mL})$ was added to the $\mathrm{NaOBu}$ solution and the mixture was stirred for $10 \mathrm{~h}$ at room temperature under a nitrogen atmosphere. The mixture was acidified with conc. $\mathrm{HCl}(75 \mathrm{~mL})$ to give a clear brown solution and an insoluble white precipitate. The solution was decanted into a separatory funnel, diluted with $\mathrm{CHCl}_{3}(600 \mathrm{~mL})$, washed with $\mathrm{H}_{2} \mathrm{O}(2 \times 500 \mathrm{~mL})$, and the solvent was evaporated under reduced pressure. Coevaporation with $\mathrm{CHCl}_{3}(2 \times 500 \mathrm{~mL})$ followed by drying under high vacuum was necessary to remove all $\mathrm{BuOH}$ to afford calixarene $6(7.0 \mathrm{~g}, 53 \%)$ as a light brown solid. ${ }^{1} \mathrm{H}$ NMR (400 MHz, $\mathrm{CDCl}_{3}$ ): $\delta 10.12$ (s, $\left.4 \mathrm{H}, \mathrm{OH}\right), 6.99$ (s, $\left.8 \mathrm{H}, \mathrm{ArH}\right), 4.25$ (s, 8 H, Ar$\mathrm{CH}_{2}-\mathrm{OBu}$ ), 4.22 (d, $4 \mathrm{H}, \mathrm{J}=13.3 \mathrm{~Hz}, \mathrm{CH}_{2}$-bridge), 3.50 (d, $4 \mathrm{H}, \mathrm{J}=13.3 \mathrm{~Hz}, \mathrm{CH}_{2}$-bridge), 3.37 (t, $\left.8 \mathrm{H}, \mathrm{J}=6.5 \mathrm{~Hz}, \mathrm{OCH}_{2} \mathrm{CH}_{2} \mathrm{CH}_{2} \mathrm{CH}_{3}\right), 1.59-1.49\left(\mathrm{~m}, 8 \mathrm{H}, \mathrm{OCH}_{2} \mathrm{CH}_{2} \mathrm{CH}_{2} \mathrm{CH}_{3}\right), 1.41-1.30(\mathrm{~m}, 8$ $\left.\mathrm{H}, \mathrm{OCH}_{2} \mathrm{CH}_{2} \mathrm{CH}_{2} \mathrm{CH}_{3}\right), 0.90\left(\mathrm{t}, 12 \mathrm{H}, \mathrm{J}=7.3 \mathrm{~Hz}, \mathrm{OCH}_{2} \mathrm{CH}_{2} \mathrm{CH}_{2} \mathrm{CH}_{3}\right) ;{ }^{13} \mathrm{C} \mathrm{NMR}(100 \mathrm{MHz}$, $\left.\mathrm{CDCl}_{3}\right): \delta 148.2,132.3,128.4,128.0,72.3,70.0,31.8,31.7,19.4,13.9 ; \mathrm{HRMS}(\mathrm{FAB})[\mathrm{M}+\mathrm{H}]^{+}$ calcd for $\mathrm{C}_{28} \mathrm{H}_{55} \mathrm{~N}_{5} \mathrm{O}_{5} \mathrm{Si}_{3} 626.3589$, found 626.3567 .

\section{5,11,17,23-Tetrakis(butoxymethyl)-25,26,27,28-tetra-(ethoxycarbonylmethoxy)}

calix[4]arene 1,3-alternate (7). Calixarene 6 (6.5 g, $8.46 \mathrm{mmol})$ was dissolved in acetone (250 $\mathrm{mL}) . \mathrm{Cs}_{2} \mathrm{CO}_{3}(13.8 \mathrm{~g}, 42.3 \mathrm{mmol})$ and ethyl bromoacetate $(4.7 \mathrm{~mL}, 42.3 \mathrm{mmol})$ was added and the resulting suspension was stirred at reflux under a nitrogen atmosphere for $24 \mathrm{~h}$. The solvent was evaporated under reduced pressure. The remaining solid was taken into $\mathrm{CH}_{2} \mathrm{Cl}_{2}(300 \mathrm{~mL})$, washed with $\mathrm{H}_{2} \mathrm{O}(2 \times 300 \mathrm{~mL})$, concentrated, and purified by flash chromatography (1 \% $\mathrm{MeOH}$ in $\mathrm{CH}_{2} \mathrm{Cl}_{2}$ ) to give calixarene 7 (4.33 g, $46 \%$ ) as a tinted oil. ${ }^{1} \mathrm{H} \mathrm{NMR} \mathrm{(400} \mathrm{MHz}$, $\left.\mathrm{CDCl}_{3}\right): \delta 7.07(\mathrm{~s}, 8 \mathrm{H}, \mathrm{ArH}), 4.29\left(\mathrm{~s}, 8 \mathrm{H}, \mathrm{Ar}-\mathrm{CH}_{2} \mathrm{-OBu}\right), 4.23\left(\mathrm{q}, 8 \mathrm{H}, \mathrm{J}=7.0 \mathrm{~Hz}, \mathrm{OCH}_{2} \mathrm{CH}_{3}\right)$, $4.00\left(\mathrm{~s}, 8 \mathrm{H}, \mathrm{CH}_{2}\right), 3.75\left(\mathrm{~s}, 8 \mathrm{H}, \mathrm{CH}_{2}\right), 3.42\left(\mathrm{t}, 8 \mathrm{H}, \mathrm{J}=6.6 \mathrm{~Hz}, \mathrm{OCH}_{2} \mathrm{CH}_{2} \mathrm{CH}_{2} \mathrm{CH}_{3}\right), 1.62-1.54$ (m, $\left.8 \mathrm{H}, \mathrm{OCH}_{2} \mathrm{CH}_{2} \mathrm{CH}_{2} \mathrm{CH}_{3}\right), 1.44-1.33\left(\mathrm{~m}, 8 \mathrm{H}, \mathrm{OCH}_{2} \mathrm{CH}_{2} \mathrm{CH}_{2} \mathrm{CH}_{3}\right), 1.32$ (t, $12 \mathrm{H}, \mathrm{J}=7.0 \mathrm{~Hz}$, $\left.\mathrm{OCH}_{2} \mathrm{CH}_{3}\right), 0.92\left(\mathrm{t}, 12 \mathrm{H}, \mathrm{J}=7.5 \mathrm{~Hz}, \mathrm{OCH}_{2} \mathrm{CH}_{2} \mathrm{CH}_{2} \mathrm{CH}_{3}\right) ;{ }^{13} \mathrm{C} \mathrm{NMR}\left(100 \mathrm{MHz}, \mathrm{CDCl}_{3}\right)$ : 
$\delta 169.3,155.1,133.2,132.6,130.3,72.7,70.1,69.4,60.7,35.9,31.9,19.4,14.2,14.0$; HRMS (FAB) $[\mathrm{M}+\mathrm{Cs}]^{+}$calcd for $\mathrm{C}_{64} \mathrm{H}_{88} \mathrm{O}_{16} \mathrm{Cs} 1245.5127$, found 1245.5179 .

\section{5,11,17,23-Tetrakis(butoxymethyl)-25,26,27,28-tetra-(hydroxycarbonylmethoxy)}

calix[4]arene 1,3-alternate (8). A solution of calixarene 7 (3.43 g, $3.10 \mathrm{mmol})$ and $45 \mathrm{wt} \%$ aqueous $\mathrm{KOH}(10 \mathrm{~mL}, 117 \mathrm{mmol})$ in $\mathrm{MeOH}(30 \mathrm{~mL})$ and THF $(30 \mathrm{~mL})$ was stirred at room temperature for $2 \mathrm{~h}$. The solvent was evaporated under reduced pressure and the remaining solid was taken into $\mathrm{H}_{2} \mathrm{O}(75 \mathrm{~mL})$. The aqueous solution was acidified to $\mathrm{pH} 1$ with $6 \mathrm{~N} \mathrm{HCl}$, extracted with $\mathrm{CH}_{2} \mathrm{Cl}_{2}(2 \times 50 \mathrm{~mL})$, and the combined organic layers were dried over $\mathrm{Na}_{2} \mathrm{SO}_{4}$ and the solvent evaporated under reduced pressure to give calixarene $8(3.0 \mathrm{~g}, 97 \%)$ as a light brown solid. ${ }^{1} \mathrm{H}$ NMR (400 MHz, $\mathrm{CDCl}_{3}$ ): $\delta 7.07$ (s, $\left.8 \mathrm{H}, \mathrm{ArH}\right), 4.34\left(\mathrm{~s}, 8 \mathrm{H}, \mathrm{CH}_{2}\right), 4.09$ (s, $8 \mathrm{H}$, $\mathrm{CH}_{2}$ ), 3.88 (s, $8 \mathrm{H}, \mathrm{CH}_{2}$ ), 3.49 (t, $\left.8 \mathrm{H}, \mathrm{J}=6.5 \mathrm{~Hz}, \mathrm{OCH}_{2} \mathrm{CH}_{2} \mathrm{CH}_{2} \mathrm{CH}_{3}\right), 1.65-1.52$ (m, $8 \mathrm{H}$, $\left.\mathrm{OCH}_{2} \mathrm{CH}_{2} \mathrm{CH}_{2} \mathrm{CH}_{3}\right), 1.43-1.30\left(\mathrm{~m}, 8 \mathrm{H}, \mathrm{OCH}_{2} \mathrm{CH}_{2} \mathrm{CH}_{2} \mathrm{CH}_{3}\right), 0.92$ (t, $12 \mathrm{H}, \mathrm{J}=7.3 \mathrm{~Hz}$, $\left.\mathrm{OCH}_{2} \mathrm{CH}_{2} \mathrm{CH}_{2} \mathrm{CH}_{3}\right) ;{ }^{13} \mathrm{C}$ NMR (100 MHz, $\left.\mathrm{CDCl}_{3}\right): \delta 168.4,152.9,135.7,133.3,129.2,72.0$, 70.9, 67.1, 37.3, 31.6, 19.3, 13.9; HRMS (FAB) $[\mathrm{M}+\mathrm{Cs}]^{+}$calcd for $\mathrm{C}_{56} \mathrm{H}_{72} \mathrm{O}_{16} \mathrm{Cs} 1133.3875$, found 1133.3827 .

\section{5,11,17,23-Tetrakis(butoxymethyl)-25,26,27,28-tetra-(chlorocarbonylmethoxy)}

calix[4]arene 1,3-alternate (9). A solution of calixarene $8(3.0 \mathrm{~g}, 3.00 \mathrm{mmol})$ and $\mathrm{SOCl}_{2}(7.44$ $\mathrm{mL}, 102 \mathrm{mmol})$ in benzene $(30 \mathrm{~mL})$ was stirred at reflux under a nitrogen atmosphere for $2 \mathrm{~h}$. The solvent was evaporated under reduced pressure and the remaining solid was coevaporated with benzene $(2 \times 30 \mathrm{~mL})$ to give calixarene $9(3.0 \mathrm{~g}, 93 \%)$ as a brownish solid. IR (neat) 1812 , 1465, 1512, 1101, $937 \mathrm{~cm}-1$; ${ }^{1} \mathrm{H}$ NMR $\left(400 \mathrm{MHz}, \mathrm{CDCl}_{3}\right): \delta 7.10(\mathrm{~s}, 8 \mathrm{H}, \mathrm{ArH}), 4.36(\mathrm{~s}, 8 \mathrm{H}$, $\mathrm{CH}_{2}$ ), 3.96 (s, $8 \mathrm{H}, \mathrm{CH}_{2}$ ), 3.94 (s, $8 \mathrm{H}, \mathrm{CH}_{2}$ ), 3.47 (t, $8 \mathrm{H}, \mathrm{J}=6.8 \mathrm{~Hz}, \mathrm{OCH}_{2} \mathrm{CH}_{2} \mathrm{CH}_{2} \mathrm{CH}_{3}$ ), 1.65 $1.56\left(\mathrm{~m}, 8 \mathrm{H}, \mathrm{OCH}_{2} \mathrm{CH}_{2} \mathrm{CH}_{2} \mathrm{CH}_{3}\right), 1.45-1.35\left(\mathrm{~m}, 8 \mathrm{H}, \mathrm{OCH}_{2} \mathrm{CH}_{2} \mathrm{CH}_{2} \mathrm{CH}_{3}\right), 0.93$ (t, $12 \mathrm{H}, \mathrm{J}=7.5$ $\mathrm{Hz}, \mathrm{OCH}_{2} \mathrm{CH}_{2} \mathrm{CH}_{2} \mathrm{CH}_{3}$ ).

2',3',5'-O-tri-tert-butyldimethylsilylguanosine (10). Guanosine 10 was prepared following the procedure of $\mathrm{Li}$ and Miller ${ }^{1}$ and recrystallized from $\mathrm{MeOH}$ to give a white solid in $80 \%$ yield. ${ }^{1} \mathrm{H}$ NMR (400 MHz, DMSO-d ${ }_{6}$ ): $\delta 10.61$ (s, 1 H, NH1), 7.88 (s, 1 H, H8), 6.45 (bs, 2 H, $\mathrm{NH}_{2}$ ),

\footnotetext{
${ }^{1}$ Li, H.; Miller, M. J. J. Org. Chem. 1999, 64, 9289.
} 
5.74 (d, 1 H, J = 7.2 Hz, H1'), 4.57 (dd, 1 H, J = 7.2, 4.4 Hz, H2'), 4.15 (d, 1 H, J = 4.4 Hz, H3'), 3.97-3.91 (m, 1 H, H4'), 3.84 (dd, 1 H, J = 11.1, 5.5 Hz, H5'), 3.70 (dd, 1 H, J = 11.1, 3.6 Hz,

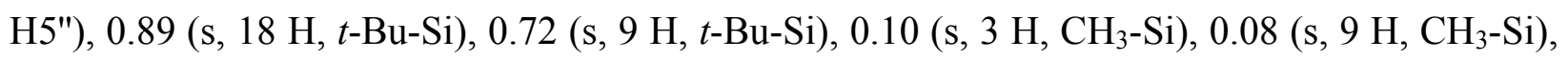
-0.09 (s, $3 \mathrm{H}, \mathrm{CH}_{3}-\mathrm{Si}$ ), -0.29 (s, $3 \mathrm{H}, \mathrm{CH}_{3}-\mathrm{Si}$ ); ${ }^{13} \mathrm{C}$ NMR (100 MHz, DMSO-d 6 ): $\delta$ 156.7, 153.8, 151.6, 134.9, 116.5, 85.7, 85.2, 75.2, 72.8, 62.9, 25.8, 25.7, 25.5, 18.0, 17.8, 17.5, -4.7, -4.8, -5.5, -5.8; HRMS (FAB) $[\mathrm{M}+\mathrm{H}]^{+}$calcd for $\mathrm{C}_{28} \mathrm{H}_{56} \mathrm{~N}_{5} \mathrm{O}_{5} \mathrm{Si}_{3} 626.3589$, found 626.3567 .

2',3'-O-di-tert-butyldimethylsilylguanosine (11). Guanosine 10 (10.0 g, 16.0 mmol)) was dissolved in nitromethane $(140 \mathrm{~mL})$. A solution of $\mathrm{ZnBr}_{2}$ in aqueous nitromethane $(140 \mathrm{~mL}, 49 \mathrm{~g}$ $\mathrm{ZnBr}_{2}$ in $350 \mathrm{~mL}$ nitromethane and $3.5 \mathrm{~mL} \mathrm{H} \mathrm{H}_{2} \mathrm{O}$ ) was added, and the mixture stirred for $4 \mathrm{~d}$ at room temperature. ${ }^{2}$ The reaction mixture was poured into $2 \mathrm{M} \mathrm{NH}_{4} \mathrm{OAc}(1 \mathrm{~L})$, extracted with $\mathrm{CH}_{2} \mathrm{Cl}_{2}(700 \mathrm{~mL})$, washed with $\mathrm{H}_{2} \mathrm{O}(700 \mathrm{~mL})$, dried over $\mathrm{Na}_{2} \mathrm{SO}_{4}$ and solvent was evaporated under reduced pressure. Crystallization from $\mathrm{MeOH}: \mathrm{CHCl}_{3}$ gave guanosine 11 (6.54 g, 80 \%) as a white solid. ${ }^{1} \mathrm{H}$ NMR (400 MHz, DMSO-d $)$ ): $\delta 10.61$ (s, 1 H, NH1), 7.96 (s, 1 H, H8), 6.42 (bs, $\left.2 \mathrm{H}, \mathrm{NH}_{2}\right), 5.71\left(\mathrm{~d}, 1 \mathrm{H}, \mathrm{J}=7.2 \mathrm{~Hz}, \mathrm{H} 1^{\prime}\right), 5.21(\mathrm{t}, 1 \mathrm{H}, \mathrm{J}=5.6 \mathrm{~Hz}, \mathrm{OH}), 4.67$ (dd, $1 \mathrm{H}, \mathrm{J}=$ 7.2, 4.4 Hz, H2'), 4.21 (d, 1 H, J = 4.4 Hz, H3'), 3.93-3.88 (m, 1 H, H4'), 3.69-3.60 (m, 1 H, H5'), 3.58-3.50 (m, 1H, H5"), 0.90 (s, 9 H, t-Bu-Si), 0.72 (s, 9 H, $t$-Bu-Si), 0.10 (s, 3 H, CH 3 -Si), 0.09 (s, $\left.3 \mathrm{H}, \mathrm{CH}_{3}-\mathrm{Si}\right) . \quad-0.10$ (s, $\left.3 \mathrm{H}, \mathrm{CH}_{3}-\mathrm{Si}\right),-0.32$ (s, $\left.3 \mathrm{H}, \mathrm{CH}_{3}-\mathrm{Si}\right) ;{ }^{13} \mathrm{C}$ NMR (100 MHz, DMSO$\left.\mathrm{d}_{6}\right): \delta 156.7,153.7,151.4,135.7,116.7,86.7,85.7,75.0,73.1,61.3,25.7,25.5,17.8,17.6,-4.7$, 4.8, -5.0, -5.5; HRMS (FAB) $[\mathrm{M}+\mathrm{H}]^{+}$calcd for $\mathrm{C}_{22} \mathrm{H}_{42} \mathrm{~N}_{5} \mathrm{O}_{5} \mathrm{Si}_{2}$ 512.2725, found 512.2737.

2',3'-O-di-tert-butyldimethylsilyl-2-N,N-dimethylaminomethyleneguanosine (12). Tо а solution of guanosine 11 (6.54 g, $12.8 \mathrm{mmol})$ in DMF (50 mL) was added DMF-dimethylacetal $(5.1 \mathrm{~mL}, 38.4 \mathrm{mmol})$ and the solution was stirred at room temperature under nitrogen for $20 \mathrm{~h}$. The reaction mixture was poured into $\mathrm{H}_{2} \mathrm{O}(100 \mathrm{~mL})$, extracted with EtOAc $(200 \mathrm{~mL})$, washed with $\mathrm{H}_{2} \mathrm{O}(2 \times 100 \mathrm{~mL})$, dried over $\mathrm{Na}_{2} \mathrm{SO}_{4}$ and the solvent was evaporated under reduced pressure to give guanosine $12(6.75 \mathrm{~g}, 93 \%)$ as a white solid. ${ }^{1} \mathrm{H}$ NMR (400 MHz, DMSO-d 6 ): $\delta 11.41$ (s, $1 \mathrm{H}, \mathrm{NH} 1), 8.46\left(\mathrm{~s}, 1 \mathrm{H}, \mathrm{N}=\mathrm{CH}-\mathrm{N}\left(\mathrm{CH}_{3}\right)_{2}\right), 8.10(\mathrm{~s}, 1 \mathrm{H}, \mathrm{H} 8), 5.78$ (d, $1 \mathrm{H}, \mathrm{J}=6.0 \mathrm{~Hz}$, H1'), 5.21 (t, 1 H, J = 5.6 Hz, OH), 4.70 (dd, 1 H, J = 6.0, 4.8 Hz, H2'), 4.31-4.27 (m, 1 H, H3'),

\footnotetext{
${ }^{2}$ Procedure described in: Seela, F.; Ott, J.; Potter, B. V. L. J. Am. Chem. Soc. 1983, 105, 5879.
} 
3.95-3.90 (m, 1 H, H4'), 3.76-3.67 (m, 1 H, H5'), 3.63-3.54 (m, 1H, H5"), 3.11 (s, 3 H, N=CH$\left.\mathrm{N}\left(\mathrm{CH}_{3}\right)_{2}\right), 3.02\left(\mathrm{~s}, 3 \mathrm{H}, \mathrm{N}=\mathrm{CH}-\mathrm{N}\left(\mathrm{CH}_{3}\right)_{2}\right), 0.90(\mathrm{~s}, 9 \mathrm{H}, t-\mathrm{Bu}-\mathrm{Si}), 0.74$ (s, $9 \mathrm{H}, t$-Bu-Si), 0.11 (s, 3 $\mathrm{H}, \mathrm{CH}_{3}-\mathrm{Si}$ ), 0.09 (s, $3 \mathrm{H}, \mathrm{CH}_{3}-\mathrm{Si}$ ). -0.09 (s, $3 \mathrm{H}, \mathrm{CH}_{3}-\mathrm{Si}$ ), - 0.30 (s, $3 \mathrm{H}, \mathrm{CH}_{3}-\mathrm{Si}$ ); ${ }^{3} \mathrm{C}$ NMR (100 MHz, DMSO-d $\left.\mathrm{d}_{6}\right): \delta 157.8,157.5,157.3,149.7,137.5,120.2,87.1,86.0,74.6,72.3,60.9,40.7$, 34.6, 25.7, 25.5, 17.8, 17.6, -4.6, -4.8, -5.5; HRMS (FAB) $[\mathrm{M}+\mathrm{H}]^{+}$calcd for $\mathrm{C}_{25} \mathrm{H}_{47} \mathrm{~N}_{6} \mathrm{O}_{5} \mathrm{Si}_{2}$ 567.3147, found 567.3144.

Note: The N,N-dimethylmethylene protecting group is necessary to prevent the formation of an iminophosphorane under Mitsunobu conditions. ${ }^{3}$

\section{5'-phthalimido-5'-deoxy-2',3'-O-di-tert-butyldimethylsilyl-2- $N, N$-dimethyl-}

aminomethyleneguanosine (13). A stirred solution of guanosine 12 (6.64 g, $11.7 \mathrm{mmol}), \mathrm{Ph}_{3} \mathrm{P}$ (3.69 $\mathrm{g}, 14.1 \mathrm{mmol})$ and phthalimide $(2.07 \mathrm{~g}, 14.1 \mathrm{mmol})$ in THF $(175 \mathrm{~mL})$ was cooled to $0^{\circ} \mathrm{C}$ under a nitrogen atmosphere. DEAD $(2.72 \mathrm{~mL}, 14.1 \mathrm{mmol})$ was added dropwise over $1 \mathrm{~min}$ and the solution was allowed to warm to room temperature and stirred for $21 \mathrm{~h}$. The solvent was evaporated under reduced pressure and the remaining oil was taken into EtOAc (200 $\mathrm{mL})$, washed with $0.1 \mathrm{~N} \mathrm{NaOH}(2 \times 100 \mathrm{~mL}), 0.1 \mathrm{~N} \mathrm{HCl}(100 \mathrm{~mL})$, saturated $\mathrm{NaCl}_{(\mathrm{aq})}(100 \mathrm{~mL})$, and dried over $\mathrm{Na}_{2} \mathrm{SO}_{4}$. After solvent evaporation, the oil was purified by flash chromatography (3\% $\mathrm{MeOH}$ in $\mathrm{CH}_{2} \mathrm{Cl}_{2}$ ) to give crude guanosine 13 (contained $\mathrm{Ph}_{3} \mathrm{P}=\mathrm{O}$ ), which was carried on without further purification. An analytical sample was obtained from the column. ${ }^{1} \mathrm{H}$ NMR (400 MHz, DMSO-d $\left.)_{6}\right): \delta 11.42(\mathrm{bs}, 1 \mathrm{H}, \mathrm{NH} 1), 8.54\left(\mathrm{~s}, 1 \mathrm{H}, \mathrm{N}=\mathrm{CH}-\mathrm{N}\left(\mathrm{CH}_{3}\right)_{2}\right), 8.10(\mathrm{~s}, 1 \mathrm{H}, \mathrm{H} 8)$, 7.95-7.89 (m, 2 H, Phthalimide-ArH), 7.88-7.82 (m, 2 H, Phthalimide-ArH), 5.80 (d, 1 H, J = 7.5 Hz, H1'), 5.39 (dd, 1 H, J = 7.5, 4.3 Hz, H2'), 4.19-4.08 (m, 3 H, H3', H4', H5'), 3.89-3.78 (m, 1 H, H5"), 3.06 (s, $\left.3 \mathrm{H}, \mathrm{N}=\mathrm{CH}-\mathrm{N}\left(\mathrm{CH}_{3}\right)_{2}\right), 3.01\left(\mathrm{~s}, 3 \mathrm{H}, \mathrm{N}=\mathrm{CH}-\mathrm{N}\left(\mathrm{CH}_{3}\right)_{2}\right), 0.79$ (s, $9 \mathrm{H}, t$-Bu-Si), $0.71\left(\mathrm{~s}, 9 \mathrm{H}, t\right.$-Bu-Si), -0.04 (s, $\left.3 \mathrm{H}, \mathrm{CH}_{3}-\mathrm{Si}\right),-0.10$ (s, $\left.6 \mathrm{H}, \mathrm{CH}_{3}-\mathrm{Si}\right) .-0.33$ (s, $\left.3 \mathrm{H}, \mathrm{CH}_{3}-\mathrm{Si}\right)$; ${ }^{13} \mathrm{C}$ NMR (100 MHz, DMSO-d 6 ): $\delta 168.1,157.6,157.5,157.1,149.8,138.9,135.0,131.3,123.4$, $120.8,87.4,82.5,73.5,72.2,40.7,34.8,25.6,25.5,17.8,17.6,-4.8,-5.1,-5.5$; HRMS (FAB) $[\mathrm{M}+\mathrm{H}]^{+}$calcd for $\mathrm{C}_{33} \mathrm{H}_{50} \mathrm{~N}_{7} \mathrm{O}_{6} \mathrm{Si}_{2} 696.3361$, found 696.3334 .

5'-phthalimido-5'-deoxy-2',3'-O-di-tert-butyldimethylsilylguanosine (14). Crude guanosine 13 was dissolved in $\mathrm{EtOH}(240 \mathrm{~mL}), \mathrm{ZnCl}_{2}(8.0 \mathrm{~g}, 58.5 \mathrm{mmol})$ was added and the suspension

\footnotetext{
${ }^{3}$ Chien, T.-C.; Chen, C.-S.; Yeh, J.-Y.; Wang, K.-C.; Chern, J.-W. Tetrahedron Lett. 1995, 36, 7881.
} 
was stirred at reflux for $16 \mathrm{~h}$. The solvent was evaporated under reduced pressure, and the remaining oil was taken into $\mathrm{CH}_{2} \mathrm{Cl}_{2}(500 \mathrm{~mL})$, washed with $\mathrm{H}_{2} \mathrm{O}(2 \times 250 \mathrm{~mL})$ and dried over $\mathrm{Na}_{2} \mathrm{SO}_{4}$. After solvent evaporation, the solid was purified by flash chromatography (3-5\% $\mathrm{MeOH}$ in $\left.\mathrm{CH}_{2} \mathrm{Cl}_{2}\right)$ to give guanosine 14 (5.1 g, 69\% over 2 steps) as a yellowish solid. ${ }^{1} \mathrm{H}$ NMR (400 MHz, DMSO-d $\left.)_{6}\right): \delta 10.65(\mathrm{~s}, 1 \mathrm{H}, \mathrm{NH} 1), 8.04(\mathrm{~s}, 1 \mathrm{H}, \mathrm{H} 8), 7.93-7.88(\mathrm{~m}, 2 \mathrm{H}$, Phthalimide-ArH), 7.87-7.82 (m, 2 H, Phthalimide-ArH), 6.43 (bs, $\left.2 \mathrm{H}, \mathrm{NH}_{2}\right), 5.72$ (d, $1 \mathrm{H}, \mathrm{J}=$ 8.0 Hz, H1'), 4.93 (dd, 1 H, J = 8.0, 4.5 Hz, H2'), 4.23 (d, 1 H, J = 4.5 Hz, H3'), 4.16-4.04 (m, 2 H, H4', H5'), 3.95-3.85 (m, 1 H, H5"), 0.80 (s, 9 H, $t$-Bu-Si), 0.71 (s, 9 H, $t$-Bu-Si), 0.01 (s, 3 H, $\left.\mathrm{CH}_{3}-\mathrm{Si}\right),-0.04\left(\mathrm{~s}, 3 \mathrm{H}, \mathrm{CH}_{3}-\mathrm{Si}\right),-0.06$ (s, $\left.3 \mathrm{H}, \mathrm{CH}_{3}-\mathrm{Si}\right),-0.32$ (s, $\left.3 \mathrm{H}, \mathrm{CH}_{3}-\mathrm{Si}\right) ;{ }^{13} \mathrm{C} \mathrm{NMR}(100$ MHz, DMSO-d ${ }_{6}$ ): $\delta 168.0,156.8,153.7,151.6,136.3,134.9,131.3,123.4,116.9,98.0,85.7$, 83.1, 73.7, 73.6, 25.6, 17.8, 17.6, -4.7, -5.1, -5.6; HRMS (FAB) $[\mathrm{M}+\mathrm{H}]^{+}$calcd for $\mathrm{C}_{30} \mathrm{H}_{45} \mathrm{~N}_{6} \mathrm{O}_{6} \mathrm{Si}_{2}$ 641.2940, found 641.2927.

5'-amino-5'-deoxy-2',3'-O-di-tert-butyldimethylsilylguanosine (15). A solution of guanosine $14(4.56 \mathrm{~g}, 7.12 \mathrm{mmol})$ and $\mathrm{H}_{2} \mathrm{NNH}_{2}(2.23 \mathrm{~mL}, 71.2 \mathrm{mmol})$ in $\mathrm{EtOH}(150 \mathrm{~mL})$ was stirred at reflux for $2.5 \mathrm{~h}$. Upon cooling to room temperature, a white solid precipitated out of solution and was collected by filtration $(700 \mathrm{mg})$. The filtrate was evaporated under reduced pressure and the remaining white solid was triturated with ether and collected (1.6 g). Combining the solids gave guanosine $15(2.3 \mathrm{~g}, 64 \%)$ as a white solid. Note: guanosine 15 was contaminated with phthalhydrazide. The reported yield was calculated by subtracting the mass of phthalhydrazide, determined by NMR integration. Phthalhydrazide does not affect the final coupling to calixarene 9, and is removed in the final purification of cG 2. An analytical sample of guanosine $\mathbf{1 5}$ was obtained by crystallization from EtOH. ${ }^{1} \mathrm{H}$ NMR (400 MHz, DMSO-d 6 ): $\delta 7.98$ (s, $\left.1 \mathrm{H}, \mathrm{H} 8\right)$, 6.56 (bs, 2 H, C2-NH 2 ), 5.69 (d, 1 H, J = 7.5 Hz, H1'), 4.76 (dd, 1 H, J = 7.5, 4.8 Hz, H2'), 4.24 (d, 1 H, J = 4.8 Hz, H3'), 3.89 (t, 1 H, J = 5.3 Hz, H4'), 2.92-2.82 (m, 2 H, H5', H5"), 0.89 (s, 9 $\mathrm{H}, t$-Bu-Si), $0.70\left(\mathrm{~s}, 9 \mathrm{H}, t\right.$-Bu-Si), $0.10\left(\mathrm{~s}, 3 \mathrm{H}, \mathrm{CH}_{3}-\mathrm{Si}\right), 0.08$ (s, $\left.3 \mathrm{H}, \mathrm{CH}_{3}-\mathrm{Si}\right),-0.10(\mathrm{~s}, 3 \mathrm{H}$, $\mathrm{CH}_{3}-\mathrm{Si}$ ), -0.36 (s, $\left.3 \mathrm{H}, \mathrm{CH}_{3}-\mathrm{Si}\right) ;{ }^{13} \mathrm{C} \mathrm{NMR}\left(100 \mathrm{MHz}, \mathrm{DMSO}-\mathrm{d}_{6}\right): \delta 156.9,153.8,151.4,136.4$, $117.0,86.9,86.1,74.1,73.1,43.1,25.8,25.5,17.8,17.5,-4.6,-4.7,-5.6$; HRMS (FAB) $[\mathrm{M}+\mathrm{H}]^{+}$ calcd for $\mathrm{C}_{22} \mathrm{H}_{43} \mathrm{~N}_{6} \mathrm{O}_{4} \mathrm{Si}_{2}$ 511.2884, found 511.2882. 
Scheme S1. Synthesis of 5'-amino-2',3'-di-tert-butyldimethylsilyladenosine 21.
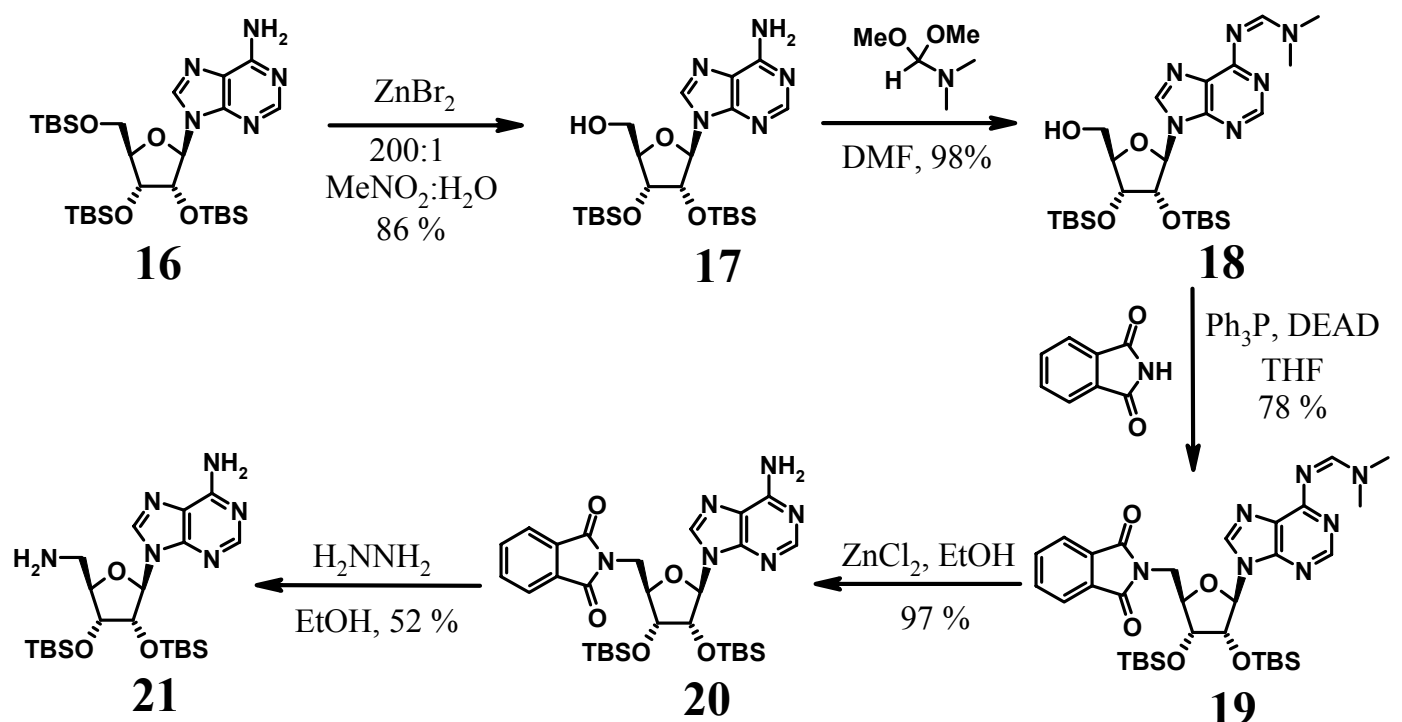

21

20

19

2',3',5'-O-tri-tert-butyldimethylsilyladenosine (16). Adenosine 16 was prepared following the procedure of $\mathrm{Li}$ and Miller ${ }^{1}$ and purified by flash chromatography $\left(2-10 \% \mathrm{MeOH}\right.$ in $\left.\mathrm{CH}_{2} \mathrm{Cl}_{2}\right)$ to give a white solid in $89 \%$ yield. ${ }^{1} \mathrm{H}$ NMR (400 MHz, DMSO-d 6 ): $\delta 8.32(\mathrm{~s}, 1 \mathrm{H}, \mathrm{H} 2), 8.12(\mathrm{~s}, 1$ H, H8), 7.31 (bs, 2 H, NH2), 5.91 (d, 1 H, J = 6.5 Hz, H1'), 4.89 (dd, 1 H, J = 6.5, 4.5 Hz, H2'), 4.30 (dd, 1 H, J = 4.5, 1.5 Hz, H3'), 4.04-3.95 (m, 2 H, H4', H5'), 3.72 (dd, 1 H, J = 13.8, 6.8 Hz,

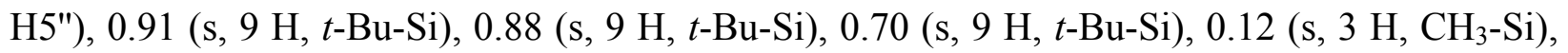
0.10 (s, $3 \mathrm{H}, \mathrm{CH}_{3}-\mathrm{Si}$ ), 0.07 (s, $6 \mathrm{H}, \mathrm{CH}_{3}-\mathrm{Si}$ ), -0.12 (s, $\left.3 \mathrm{H}, \mathrm{CH}_{3}-\mathrm{Si}\right),-0.37$ (s, $3 \mathrm{H}, t$-Bu-Si); ${ }^{13} \mathrm{C}$ NMR (100 MHz, DMSO-d 6 ): $\delta 156.1,152.5,149.4,139.5,119.2,86.8,85.2,74.2,72.3,62.5$, $25.8,25.7,25.4,18.0,17.8,17.5,-4.7,-4.8,-4.9,-5.5,-5.6$; HRMS (FAB) $[\mathrm{M}+\mathrm{H}]^{+}$calcd for $\mathrm{C}_{28} \mathrm{H}_{56} \mathrm{~N}_{5} \mathrm{O}_{4} \mathrm{Si}_{3} 610.3640$, found 610.3662 .

2',3'-O-di-tert-butyldimethylsilyladenosine (17). Adenosine 16 (3.95 g, $6.48 \mathrm{mmol}$ ) was dissolved in nitromethane $(56 \mathrm{~mL})$, a solution of $\mathrm{ZnBr}_{2}$ in aqueous nitromethane $(56 \mathrm{~mL}, 49 \mathrm{~g}$ $\mathrm{ZnBr}_{2}$ in $350 \mathrm{~mL}$ nitromethane and $3.5 \mathrm{~mL} \mathrm{H}_{2} \mathrm{O}$ ) was added, and the mixture was stirred for $4 \mathrm{~d}$ at room temperature. ${ }^{2}$ The reaction mixture was poured into $1 \mathrm{M} \mathrm{NH}_{4} \mathrm{OAc}(400 \mathrm{~mL})$, extracted with $\mathrm{CH}_{2} \mathrm{Cl}_{2}(400 \mathrm{~mL})$, washed with $\mathrm{H}_{2} \mathrm{O}(400 \mathrm{~mL})$, dried over $\mathrm{Na}_{2} \mathrm{SO}_{4}$ and the solvent was evaporated under reduced pressure. Crystallization from $\mathrm{MeOH}: \mathrm{CHCl}_{3}$ gave adenosine 17 (2.75 $\mathrm{g}, 86 \%$ ) as a white solid. ${ }^{1} \mathrm{H}$ NMR (400 MHz, DMSO-d $): \delta 8.39$ (s, $\left.1 \mathrm{H}, \mathrm{H} 2\right), 8.13(\mathrm{~s}, 1 \mathrm{H}$, 
H8), 7.39 (bs, 2 H, NH ), 5.89 (d, 1 H, J = 7.3 Hz, H1'), 5.73 (dd, 1 H, J = 8.0, 4.0 Hz, OH), 4.89 (dd, 1 H, J = 7.3, 4.5 Hz, H2'), 4.28 (dd, 1 H, J = 4.5, 0.8 Hz, H3'), 4.00-3.95 (m, 1 H, H4'), 3.72 (dt, $1 \mathrm{H}, \mathrm{J}=12.3,4.0 \mathrm{~Hz}, \mathrm{H} 5$ '), 3.62-3.52 (m, $1 \mathrm{H}, \mathrm{H} 5 "), 0.91$ (s, $9 \mathrm{H}, t$-Bu-Si), 0.69 (s, 9 H, $t$ Bu-Si), $0.11\left(\mathrm{~s}, 3 \mathrm{H}, \mathrm{CH}_{3}-\mathrm{Si}\right), 0.10$ (s, $\left.3 \mathrm{H}, \mathrm{CH}_{3}-\mathrm{Si}\right),-0.16$ (s, $\left.3 \mathrm{H}, \mathrm{CH}_{3}-\mathrm{Si}\right),-0.48$ (s, $3 \mathrm{H}, \mathrm{CH}_{3}-$ $\mathrm{Si}) ;{ }^{13} \mathrm{C}$ NMR (100 MHz, DMSO-d 6 ): $\delta$ 156.3, 152.4, 149.0, 140.4, 119.6, 87.7, 87.3, 74.2, 73.2, 61.3, 25.8, 25.5, 17.9, 17.6, -4.6, -4.7, -4.8, -5.7; HRMS (FAB) $[\mathrm{M}+\mathrm{H}]^{+}$calcd for $\mathrm{C}_{22} \mathrm{H}_{42} \mathrm{~N}_{5} \mathrm{O}_{4} \mathrm{Si}_{2}$ 496.2775, found 496.2780 .

2',3'-O-di-tert-butyldimethylsilyl-6-N,N-dimethylaminomethyleneadenosine (18). Tо a solution of adenosine $17(2.58 \mathrm{~g}, 5.21 \mathrm{mmol})$ in DMF (25 mL) was added DMF-dimethylacetal $(2.1 \mathrm{~mL}, 15.6 \mathrm{mmol})$ and the solution was stirred at room temperature under nitrogen atmosphere for $20 \mathrm{~h}$. The reaction mixture was poured into $\mathrm{H}_{2} \mathrm{O}(50 \mathrm{~mL})$, extracted with EtOAc $(75 \mathrm{~mL})$, washed with $\mathrm{H}_{2} \mathrm{O}(2 \times 50 \mathrm{~mL})$, dried over $\mathrm{Na}_{2} \mathrm{SO}_{4}$ and the solvent was evaporated under reduced pressure to give adenosine 18 (2.81 g, $98 \%$ ) as a white solid. ${ }^{1} \mathrm{H}$ NMR (400 MHz, DMSO-d 6 ): $\delta 8.92\left(\mathrm{~s}, 1 \mathrm{H}, \mathrm{N}=\mathrm{CH}-\mathrm{N}\left(\mathrm{CH}_{3}\right)_{2}\right), 8.52(\mathrm{~s}, 1 \mathrm{H}, \mathrm{H} 2), 8.41(\mathrm{~s}, 1 \mathrm{H}, \mathrm{H} 8), 5.95(\mathrm{~d}, 1 \mathrm{H}, \mathrm{J}=7.0 \mathrm{~Hz}$, H1'), 5.55 (dd, 1 H, J = 7.3, 4.5 Hz, OH), 4.90 (dd, 1 H, J = 7.0, 4.5 Hz, H2'), 4.30 (dd, 1 H, J = 4.5, 1.0 Hz, H3'), 4.00-3.96 (m, 1 H, H4'), 3.74 (dt, 1 H, J = 12.3, 4.5 Hz, H5'), 3.63-3.54 (m, 1 H, H5"), 3.19 (s, $\left.3 \mathrm{H}, \mathrm{N}=\mathrm{CH}-\mathrm{N}\left(\mathrm{CH}_{3}\right)_{2}\right), 3.13$ (s, $\left.3 \mathrm{H}, \mathrm{N}=\mathrm{CH}-\mathrm{N}\left(\mathrm{CH}_{3}\right)_{2}\right), 0.91$ (s, $9 \mathrm{H}, t$-Bu-Si), 0.68 (s, $9 \mathrm{H}, t$-Bu-Si), 0.12 (s, $3 \mathrm{H}, \mathrm{CH}_{3}-\mathrm{Si}$ ), 0.10 (s, $3 \mathrm{H}, \mathrm{CH}_{3}-\mathrm{Si}$ ), -0.16 (s, $3 \mathrm{H}, \mathrm{CH}_{3}-\mathrm{Si}$ ), -0.49 (s, $\left.3 \mathrm{H}, \mathrm{CH}_{3}-\mathrm{Si}\right) ;{ }^{13} \mathrm{C}$ NMR (100 MHz, DMSO-d 6 ): $\delta 159.4,158.2,151.8,151.1,141.9,126.1$, $87.5,87.1,74.3,73.0,61.4,40.8,34.7,25.8,25.5,17.9,17.5,-4.6,-4.7,-4.8,-5.6$; HRMS (FAB) $[\mathrm{M}+\mathrm{H}]^{+}$calcd for $\mathrm{C}_{25} \mathrm{H}_{47} \mathrm{~N}_{6} \mathrm{O}_{4} \mathrm{Si}_{2}$ 551.3198, found 551.3201.

Note: The N,N-dimethylmethylene protecting group is necessary to prevent the formation of an iminophosphorane under Mitsunobu conditions. ${ }^{3}$

\section{5'-phthalimido-5'-deoxy-2',3'-O-di-tert-butyldimethylsilyl-6- $N, N$-dimethyl-}

aminomethyleneadenosine (19). A stirred solution of adenosine 18 (2.72 g, 4.94 mmol), $\mathrm{Ph}_{3} \mathrm{P}$ $(1.55 \mathrm{~g}, 5.93 \mathrm{mmol})$ and phthalimide $(0.87 \mathrm{~g}, 5.93 \mathrm{mmol})$ in THF $(80 \mathrm{~mL})$ was cooled to $0^{\circ} \mathrm{C}$ under a nitrogen atmosphere. DEAD $(0.93 \mathrm{~mL}, 5.93 \mathrm{mmol})$ was added dropwise over $1 \mathrm{~min}$ and the solution was allowed to warm to room temperature and stirred for $21 \mathrm{~h}$. The solvent was evaporated under reduced pressure and the remaining oil was taken into EtOAc (100 $\mathrm{mL})$, 
washed with $0.1 \mathrm{~N} \mathrm{NaOH}(2 \times 50 \mathrm{~mL}), 0.1 \mathrm{~N} \mathrm{HCl}(50 \mathrm{~mL})$, saturated $\operatorname{NaCl}_{(\mathrm{aq})}(50 \mathrm{~mL})$, and dried over $\mathrm{Na}_{2} \mathrm{SO}_{4}$. After solvent evaporation, the oil was dissolved in hot $\mathrm{MeOH}$, the product crystallized upon standing overnight to give adenosine $19(2.62 \mathrm{~g}, 78 \%)$ as a white solid. ${ }^{1} \mathrm{H}$ NMR (400 MHz, DMSO-d 6 ): $\delta 8.91\left(\mathrm{~s}, 1 \mathrm{H}, \mathrm{N}=\mathrm{CH}-\mathrm{N}\left(\mathrm{CH}_{3}\right)_{2}\right), 8.59(\mathrm{~s}, 1 \mathrm{H}, \mathrm{H} 2), 8.25(\mathrm{~s}, 1 \mathrm{H}$, H8), 7.91-7.82 (m, 4 H, Phthalimide-ArH), 5.97 (d, 1 H, J = 7.5 Hz, H1'), 5.23 (dd, 1 H, J = 7.5, $\left.4.3 \mathrm{~Hz}, \mathrm{H} 2^{\prime}\right), 4.32$ (d, 1 H, J = 4.3 Hz, H3'), 4.19-4.11 (m, 2 H, H4', H5'), 3.92 (dd, 1 H, J = 17.1, $9.3 \mathrm{~Hz}, \mathrm{H} 5 "), 3.19$ (s, $\left.3 \mathrm{H}, \mathrm{N}=\mathrm{CH}-\mathrm{N}\left(\mathrm{CH}_{3}\right)_{2}\right), 3.13$ (s, $\left.3 \mathrm{H}, \mathrm{N}=\mathrm{CH}-\mathrm{N}\left(\mathrm{CH}_{3}\right)_{2}\right), 0.83$ (s, $9 \mathrm{H}, t$-BuSi), 0.65 (s, $9 \mathrm{H}, t$-Bu-Si), 0.02 (s, $3 \mathrm{H}, \mathrm{CH}_{3}-\mathrm{Si}$ ), -0.02 (s, $\left.3 \mathrm{H}, \mathrm{CH}_{3}-\mathrm{Si}\right),-0.12$ (s, $3 \mathrm{H}, \mathrm{CH}_{3}-\mathrm{Si}$ ), 0.49 (s, $3 \mathrm{H}, \mathrm{CH}_{3}-\mathrm{Si}$ ); ${ }^{13} \mathrm{C}$ NMR (100 MHz, DMSO-d 6 ): $\delta 168.1,159.3,158.1,151.9,151.6$, $142.4,134.9,131.5,126.0,123.4,86.8,83.3,73.5,72.9,40.8,34.7,25.6,25.5,17.9,17.5,-4.7$, 5.0, -5.6; HRMS (FAB) $[\mathrm{M}+\mathrm{H}]^{+}$calcd for $\mathrm{C}_{33} \mathrm{H}_{50} \mathrm{~N}_{7} \mathrm{O}_{5} \mathrm{Si}_{2} 680.3412$, found 680.3435.

5'-phthalimido-5'-deoxy-2',3'-O-di-tert-butyldimethylsilyladenosine (20). Adenosine 19 (2.51 g, $3.78 \mathrm{mmol}$ ) was dissolved in $\mathrm{EtOH}(75 \mathrm{~mL}), \mathrm{ZnCl}_{2}(1.55 \mathrm{~g}, 11.3 \mathrm{mmol})$ was added and the suspension was stirred at reflux for $1 \mathrm{~h}$. The reaction mixture was poured into $\mathrm{H}_{2} \mathrm{O}(250 \mathrm{~mL})$ and extracted with $\mathrm{CH}_{2} \mathrm{Cl}_{2}(3 \times 125 \mathrm{~mL})$. The combined organic layers were washed with saturated $\mathrm{NaCl}_{(\mathrm{aq})}(100 \mathrm{~mL})$, dried over $\mathrm{Na}_{2} \mathrm{SO}_{4}$ and the solvent was evaporated under reduced pressure to give adenosine $20\left(2.3 \mathrm{~g}, 97 \%\right.$ ) as a white solid. ${ }^{1} \mathrm{H}$ NMR (400 MHz, DMSO-d 6 ): $\delta$ 8.47 (s, 1 H, H2), 7.98 (s, 1 H, H8), 7.92-7.81 (m, 4 H, Phthalimide-ArH), 7.30 (bs, 2 H, NH ), $5.91\left(\mathrm{~d}, 1 \mathrm{H}, \mathrm{J}=7.5 \mathrm{~Hz}, \mathrm{H} 1^{\prime}\right), 5.22$ (dd, 1 H, J = 7.5, 4.5 Hz, H2'), 4.31 (d, 1 H, J = 4.5 Hz, H3'), 4.19-4.10 (m, 2 H, H4', H5'), 3.94-3.85 (m, 1 H, H5"), 0.82 (s, 9 H, $t$-Bu-Si), 0.66 (s, 9 H, $t$-Bu$\mathrm{Si}$ ), 0.02 (s, $3 \mathrm{H}, \mathrm{CH}_{3}-\mathrm{Si}$ ), -0.03 (s, $\left.3 \mathrm{H}, \mathrm{CH}_{3}-\mathrm{Si}\right),-0.11$ (s, $3 \mathrm{H}, \mathrm{CH}_{3}-\mathrm{Si}$ ), -0.45 (s, $3 \mathrm{H}, \mathrm{CH}_{3}-\mathrm{Si}$ ); ${ }^{13} \mathrm{C}$ NMR (100 MHz, DMSO-d 6 ): $\delta 168.1,156.1,152.6,149.5,140.6,134.8,131.5,123.3$, $119.4,86.8,83.1,73.5,72.9,25.6,25.5,17.8,17.5,-4.7,-4.8,-5.0,-5.6$; HRMS (FAB) $[\mathrm{M}+\mathrm{H}]^{+}$ calcd for $\mathrm{C}_{30} \mathrm{H}_{45} \mathrm{~N}_{6} \mathrm{O}_{5} \mathrm{Si}_{2} 625.2990$, found 625.3023.

5'-amino-5'-deoxy-2',3'-O-di-tert-butyldimethylsilyladenosine (21). A solution of adenosine 20 (2.08 g, $3.41 \mathrm{mmol})$ and $\mathrm{H}_{2} \mathrm{NNH}_{2}(1.2 \mathrm{~mL}, 38.3 \mathrm{mmol})$ in $\mathrm{EtOH}(60 \mathrm{~mL})$ was stirred at reflux for $2.5 \mathrm{~h}$. The solvent was evaporated under reduced pressure and the remaining solid was triturated with $\mathrm{CH}_{3} \mathrm{CN}$ to give adenosine 21 (880 mg, $52 \%$ ) as an off-white solid. An analytical 
sample was obtained by crystallization from $\mathrm{CH}_{3} \mathrm{CN} /$ hexanes. ${ }^{1} \mathrm{H}$ NMR (400 MHz, DMSO- $\mathrm{d}_{6}$ ): $\delta 8.41$ (s, $1 \mathrm{H}, \mathrm{H} 2), 8.12$ (s, $1 \mathrm{H}, \mathrm{H} 8), 7.28$ (bs, $2 \mathrm{H}, \mathrm{C} 6-\mathrm{NH}_{2}$ ), 5.85 (d, $1 \mathrm{H}, \mathrm{J}=7.3 \mathrm{~Hz}, \mathrm{H1}$ )), 4.97 (dd, $1 \mathrm{H}, \mathrm{J}=7.3,4.5 \mathrm{~Hz}, \mathrm{H} 2$ '), 4.31 (dd, $1 \mathrm{H}, \mathrm{J}=4.5,1.0 \mathrm{~Hz}, \mathrm{H3}$ '), 3.92-3.86 (m, $\left.1 \mathrm{H}, \mathrm{H} 4{ }^{\prime}\right), 2.86$

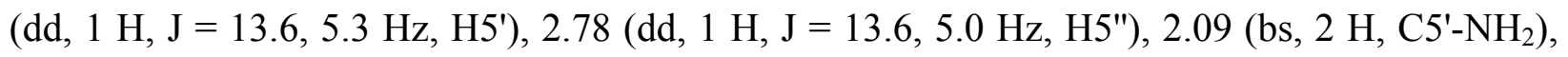
0.91 (s, $9 \mathrm{H}, t-\mathrm{Bu}-\mathrm{Si}$ ), 0.67 (s, $9 \mathrm{H}, t$-Bu-Si), 0.12 (s, $3 \mathrm{H}, \mathrm{CH}_{3}-\mathrm{Si}$ ), 0.10 (s, $3 \mathrm{H}, \mathrm{CH}_{3}-\mathrm{Si}$ ), -0.13 (s, $3 \mathrm{H}, \mathrm{CH}_{3}-\mathrm{Si}$ ), -0.48 (s, $3 \mathrm{H}, \mathrm{CH}_{3}-\mathrm{Si}$ ); ${ }^{13} \mathrm{C}$ NMR (100 MHz, DMSO-d 6 ): $\delta 156.1,152.5,149.4$, 140.6, 119.5, 87.8, 87.1, 73.6, 72.9, 43.6, 25.8, 25.5, 17.8, 17.5, -4.6, -4.7, -5.7; HRMS (FAB) $[\mathrm{M}+\mathrm{H}]^{+}$calcd for $\mathrm{C}_{22} \mathrm{H}_{43} \mathrm{~N}_{6} \mathrm{O}_{3} \mathrm{Si}_{2} 495.2935$, found 495.2902 .

Calix[4]arene-guanosine cG 2. To a solution of calixarene 9 (977 $\mathrm{mg}, 0.911 \mathrm{mmol})$ and guanosine 15 (2.79 g, $5.47 \mathrm{mmol})$ in $\mathrm{CH}_{2} \mathrm{Cl}_{2}(150 \mathrm{~mL})$ under nitrogen atmosphere was added $\mathrm{Et}_{3} \mathrm{~N}(2.5 \mathrm{~mL}, 18.2 \mathrm{mmol})$ and the mixture was stirred at room temperature for $24 \mathrm{~h}$. The solvent was evaporated under reduced pressure followed by purification by flash chromatography ( $5 \%$ $\mathrm{MeOH}$ in $\left.\mathrm{CH}_{2} \mathrm{Cl}_{2}\right)$ to give cG $2(1.34 \mathrm{~g}, 49 \%)$ as a white solid. ${ }^{1} \mathrm{H}$ NMR (400 MHz, DMSO- $\mathrm{d}_{6}$, $50{ }^{\circ} \mathrm{C}$ ): $\delta 10.52(\mathrm{~s}, 4 \mathrm{H}, \mathrm{N} 1 \mathrm{H}), 7.95$ (s, $\left.4 \mathrm{H}, \mathrm{H} 8\right), 7.93$ (bs, $4 \mathrm{H}, 5$ '-Amide NH), 7.00 (s, $4 \mathrm{H}$, Calix-ArH), 6.93 (s, 4 H, Calix-ArH), 6.06 (bs, 8 H, C2-NH ) $^{2}, 5.75$ (d, 4 H, J = 7.2 Hz, H1'), 4.76 (dd, 4 H, J = 7.2, 4.6 Hz, H2'), 4.26 (d, 4 H, J = 4.6 Hz, H3'), 4.14 (d, 4 H, J = 11.6 Hz, $\left.\mathrm{C}(\mathrm{O})-\mathrm{CH}_{2}-\mathrm{O}\right), 4.09$ (d, $4 \mathrm{H}, \mathrm{J}=11.6 \mathrm{~Hz}, \mathrm{C}(\mathrm{O})-\mathrm{CH}_{2}-\mathrm{O}$ ), 3.99 (t, $4 \mathrm{H}, \mathrm{J}=5.9 \mathrm{~Hz}, \mathrm{H} 4$ '), 3.71 (app d, $8 \mathrm{H}, \mathrm{J}=7.0 \mathrm{~Hz}, \mathrm{CH}_{2}$-bridge), 3.67-3.57 (m, $4 \mathrm{H}, \mathrm{H} 5$ '), 3.55-3.41 (m, $12 \mathrm{H}, \mathrm{H} 5 "$ + Ar- $\mathrm{CH}_{2}-\mathrm{O}$ ), $3.24\left(\mathrm{t}, 8 \mathrm{H}, \mathrm{J}=6.6 \mathrm{~Hz}, \mathrm{OCH}_{2} \mathrm{CH}_{2} \mathrm{CH}_{2} \mathrm{CH}_{3}\right), 1.49-1.37\left(\mathrm{~m}, 8 \mathrm{H}, \mathrm{OCH}_{2} \mathrm{CH}_{2} \mathrm{CH}_{2} \mathrm{CH}_{3}\right), 1.32-1.19$ $\left(\mathrm{m}, 8 \mathrm{H}, \mathrm{OCH}_{2} \mathrm{CH}_{2} \mathrm{CH}_{2} \mathrm{CH}_{3}\right), 0.91(\mathrm{~s}, 36 \mathrm{H}, t-\mathrm{Bu}-\mathrm{Si}), 0.82(\mathrm{t}, 12 \mathrm{H}, \mathrm{J}=7.4 \mathrm{~Hz}$, $\mathrm{OCH}_{2} \mathrm{CH}_{2} \mathrm{CH}_{2} \mathrm{CH}_{3}$ ), 0.75 (s, $\left.36 \mathrm{H}, t-\mathrm{Bu}-\mathrm{Si}\right), 0.11$ (s, $\left.12 \mathrm{H}, \mathrm{CH}_{3}-\mathrm{Si}\right), 0.09$ (s, $12 \mathrm{H}, \mathrm{CH}_{3}-\mathrm{Si}$ ), 0.07 (s, $12 \mathrm{H}, \mathrm{CH}_{3}-\mathrm{Si}$ ), -0.27 (s, $12 \mathrm{H}, \mathrm{CH}_{3}-\mathrm{Si}$ ); ${ }^{13} \mathrm{C} \mathrm{NMR}\left(100 \mathrm{MHz}, \mathrm{DMSO}-\mathrm{d}_{6}, 22{ }^{\circ} \mathrm{C}\right): \delta 168.8$, 156.7, 154.9, 153.5, 151.4, 136.5, 134.0, 133.5, 133.1, 130.2, 130.0, 117.1, 85.9, 84.6, 74.0, 73.8, $71.7,70.6,69.1,31.2,25.7,25.5,18.9,17.7,17.5,13.8,-4.8,-5.6$; MS (ESI) $[(\mathrm{M}+2 \mathrm{H}) / 2]^{2+}$ calcd for $\mathrm{C}_{144} \mathrm{H}_{234} \mathrm{~N}_{24} \mathrm{O}_{28} \mathrm{Si}_{8} 1486.8$, found 1486.8. Anal. calcd for $\mathrm{C}_{144} \mathrm{H}_{232} \mathrm{~N}_{24} \mathrm{O}_{28} \mathrm{Si}_{8}$ : C, 58.19; $\mathrm{H}$, 7.87, found: C, 58.05; H, 7.99. 
Scheme S2. Final coupling to give calix[4]arene-adenosine cA 3.

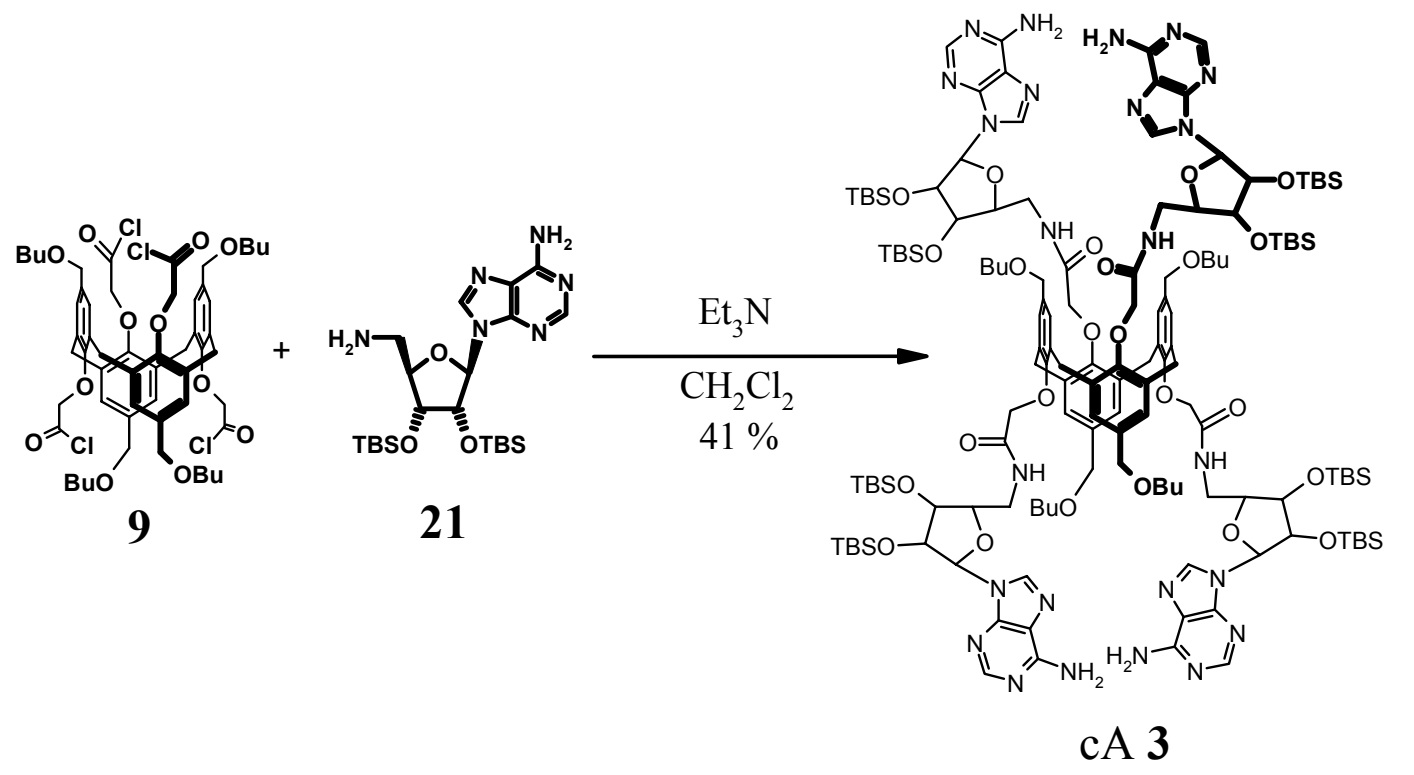

Calix[4]arene-adenosine cA 3. To a suspension of calixarene 9 (254 mg, $0.237 \mathrm{mmol})$ and adenosine 21 (680 mg, $1.42 \mathrm{mmol})$ in $\mathrm{CH}_{2} \mathrm{Cl}_{2}(20 \mathrm{~mL})$ under nitrogen atmosphere was added $\mathrm{Et}_{3} \mathrm{~N}(0.66 \mathrm{~mL}, 4.73 \mathrm{mmol})$ and the mixture was stirred at room temperature for $24 \mathrm{~h}$. The solvent was evaporated under reduced pressure followed by purification by flash chromatography ( $3 \% \mathrm{MeOH}$ in $\mathrm{CH}_{2} \mathrm{Cl}_{2}$ ) to give cA 3 (280 mg, $41 \%$ ) as a white solid. ${ }^{1} \mathrm{H}$ NMR (400 MHz, DMSO-d 6 , $50{ }^{\circ} \mathrm{C}$ ): $\delta 8.36$ (s, 4 H, H2), 8.09 (s, 4 H, H8), 7.72 (t, 4 H, J = 5.7 Hz, $5^{\prime}-$ Amide NH), 7.06 (bs, 8 H, C6-NH2), 7.01 (s, 4 H, Calix-ArH), 6.94 (s, 4 H, Calix-ArH), 5.90 (d, 4 H, J = 6.8 Hz, H1'), 5.05 (dd, 4 H, J = 6.8, 4.6 Hz, H2'), 4.37 (d, 4 H, J = 4.6 Hz, H3'), 4.17 (d, $\left.4 \mathrm{H}, \mathrm{J}=11.6 \mathrm{~Hz}, \mathrm{C}(\mathrm{O})-\mathrm{CH}_{2}-\mathrm{O}\right), 4.12\left(\mathrm{~d}, 4 \mathrm{H}, \mathrm{J}=11.6 \mathrm{~Hz}, \mathrm{C}(\mathrm{O})-\mathrm{CH}_{2}-\mathrm{O}\right), 4.01$ (t, $4 \mathrm{H}, \mathrm{J}=5.9 \mathrm{~Hz}$, H4'), 3.78-3.64 (m, 12 H, H5' + $\mathrm{CH}_{2}$-bridge), 3.55-3.35 (m, $12 \mathrm{H}, \mathrm{H} 5 "$ + Ar- $\left.\mathrm{CH}_{2}-\mathrm{O}\right), 3.25$ (t, 8 H, $\left.\mathrm{J}=6.6 \mathrm{~Hz}, \mathrm{OCH}_{2} \mathrm{CH}_{2} \mathrm{CH}_{2} \mathrm{CH}_{3}\right), 1.48-1.37\left(\mathrm{~m}, 8 \mathrm{H}, \mathrm{OCH}_{2} \mathrm{CH}_{2} \mathrm{CH}_{2} \mathrm{CH}_{3}\right), 1.31-1.19(\mathrm{~m}, 8 \mathrm{H}$, $\mathrm{OCH}_{2} \mathrm{CH}_{2} \mathrm{CH}_{2} \mathrm{CH}_{3}$ ), 0.90 (s, $36 \mathrm{H}, t$-Bu-Si), 0.81 (t, $\left.12 \mathrm{H}, \mathrm{J}=7.4 \mathrm{~Hz}, \mathrm{OCH}_{2} \mathrm{CH}_{2} \mathrm{CH}_{2} \mathrm{CH}_{3}\right), 0.70$ (s, $36 \mathrm{H}, t$-Bu-Si), 0.09 (s, $\left.12 \mathrm{H}, \mathrm{CH}_{3}-\mathrm{Si}\right), 0.07$ (s, $\left.12 \mathrm{H}, \mathrm{CH}_{3}-\mathrm{Si}\right),-0.12$ (s, $\left.12 \mathrm{H}, \mathrm{CH}_{3}-\mathrm{Si}\right),-0.41$ (s, $\left.12 \mathrm{H}, \mathrm{CH}_{3}-\mathrm{Si}\right) ;{ }^{13} \mathrm{C}$ NMR $\left(100 \mathrm{MHz}, \mathrm{DMSO}-\mathrm{d}_{6}, 22{ }^{\circ} \mathrm{C}\right): \delta 168.8,156.1,154.9,152.4,149.3$, $140.7,133.8,133.5,132.7,130.2,130.1,119.7,87.1,84.7,73.7,73.2,71.8,70.6,69.0,31.3$, 25.7, 25.6, 25.5, 18.9, 17.7, 17.5, 13.8, 0.2, -4.8, -5.7; HRMS (FAB) $[\mathrm{M}+\mathrm{Cs}]^{+}$calcd for $\mathrm{C}_{144} \mathrm{H}_{232} \mathrm{~N}_{24} \mathrm{O}_{24} \mathrm{Si}_{8} \mathrm{Cs} 3038.4880$, found 3038.4922. 
Scheme S3. Synthesis of G 4.

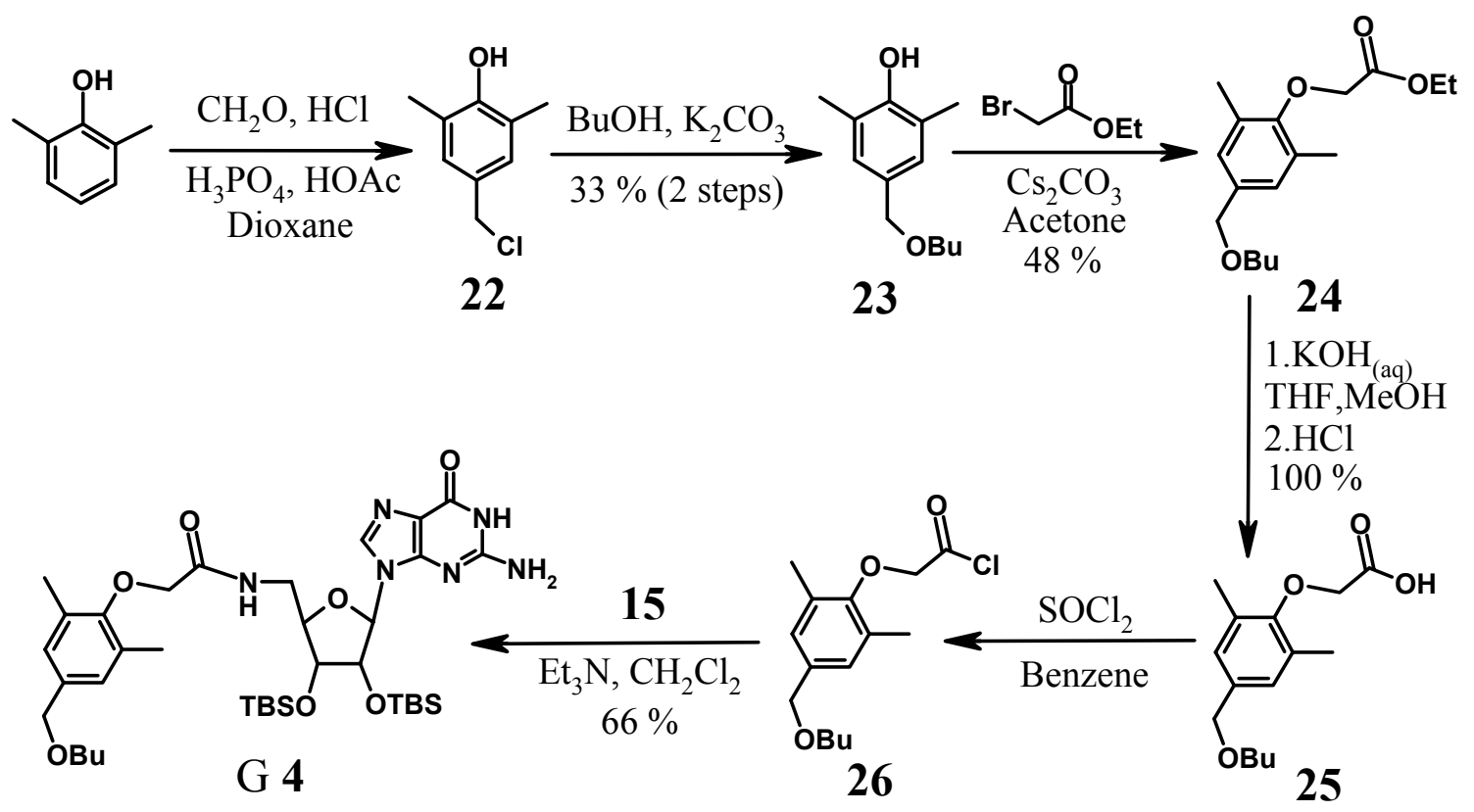

4-butoxymethyl-2,6-dimethylphenol (23). A solution of 2,6-dimethylphenol (1 g, $8.18 \mathrm{mmol})$, paraformaldehyde $(2.45 \mathrm{~g}, 81.8 \mathrm{mmol})$, glacial HOAc $(8 \mathrm{~mL})$, conc. $\mathrm{H}_{3} \mathrm{PO}_{4}(8.5 \mathrm{~mL})$, and conc. $\mathrm{HCl}(9.0 \mathrm{~mL})$ in dioxane $(85 \mathrm{~mL})$ was stirred at $80{ }^{\circ} \mathrm{C}$ for $24 \mathrm{~h}$. The orange solution was diluted with an ice/water mixture $(60 \mathrm{~mL})$ and extracted with $\mathrm{CHCl}_{3}(2 \times 70 \mathrm{~mL})$. The combined organic layers were dried over $\mathrm{Na}_{2} \mathrm{SO}_{4}$ and the solvent evaporated under reduced pressure to give crude 4-chloromethyl-2,6-dimethylphenol 22 as an orange syrup. Compound 22 was dissolved in $\mathrm{BuOH}(75 \mathrm{~mL}), \mathrm{K}_{2} \mathrm{CO}_{3}(5.66 \mathrm{~g}, 40.9 \mathrm{mmol})$ was added, and the suspension was stirred at $80{ }^{\circ} \mathrm{C}$ under a nitrogen atmosphere for $2 \mathrm{~d}$. The mixture was filtered to remove $\mathrm{K}_{2} \mathrm{CO}_{3}$ and the filtrate was diluted with $\mathrm{CHCl}_{3}(500 \mathrm{~mL})$, washed with $\mathrm{H}_{2} \mathrm{O}(2 \times 500 \mathrm{~mL})$, and concentrated to give a reddish-brown syrup. Purification by flash chromatography $\left(\mathrm{CH}_{2} \mathrm{Cl}_{2}\right)$ afforded 4-butoxymethyl2,6-dimethylphenol 23 (0.56 g, $33 \%$ over 2 steps) as a white solid. ${ }^{1} \mathrm{H}$ NMR (400 $\left.\mathrm{MHz}, \mathrm{CDCl}_{3}\right)$ : $\delta 6.96(\mathrm{~s}, 2 \mathrm{H}, \mathrm{ArH}), 4.36\left(\mathrm{~s}, 2 \mathrm{H}, \mathrm{Ar}-\mathrm{CH}_{2}-\mathrm{O}\right), 3.45$ (t, $\left.2 \mathrm{H}, \mathrm{J}=6.6 \mathrm{~Hz}, \mathrm{OCH}_{2} \mathrm{CH}_{2} \mathrm{CH}_{2} \mathrm{CH}_{3}\right), 2.24$ $\left(\mathrm{s}, 6 \mathrm{H}, \mathrm{Ar}-\mathrm{CH}_{3}\right), \quad 1.64-1.55\left(\mathrm{~m}, 2 \mathrm{H}, \quad \mathrm{OCH}_{2} \mathrm{CH}_{2} \mathrm{CH}_{2} \mathrm{CH}_{3}\right), 1.44-1.34$ (m, $2 \mathrm{H}$, $\left.\mathrm{OCH}_{2} \mathrm{CH}_{2} \mathrm{CH}_{2} \mathrm{CH}_{3}\right), 0.92$ (t, $\left.3 \mathrm{H}, \mathrm{J}=7.5 \mathrm{~Hz}, \mathrm{OCH}_{2} \mathrm{CH}_{2} \mathrm{CH}_{2} \mathrm{CH}_{3}\right) ;{ }^{13} \mathrm{C} \mathrm{NMR}\left(100 \mathrm{MHz}, \mathrm{CDCl}_{3}\right)$ : $\delta 133.4,128.8,128.4,122.8,72.7,70.0,31.8,19.4,15.9,13.9$. 
Ethyl-(4-butoxymethyl-2,6-dimethyl)-phenoxyacetate (24). Compound 23 (0.56 g, 2.69 mmol) was dissolved in acetone $(80 \mathrm{~mL}) . \mathrm{Cs}_{2} \mathrm{CO}_{3}(4.38 \mathrm{~g}, 13.5 \mathrm{mmol})$ and ethyl bromoacetate (0.45 mL, $4.03 \mathrm{mmol})$ was added and the resulting suspension was stirred at reflux under a nitrogen atmosphere for $24 \mathrm{~h}$. The solvent was evaporated under reduced pressure. The remaining oil was taken into $\mathrm{CH}_{2} \mathrm{Cl}_{2}(50 \mathrm{~mL})$, washed with $\mathrm{H}_{2} \mathrm{O}(2 \times 50 \mathrm{~mL})$, concentrated, and purified by flash chromatography $\left(\mathrm{CH}_{2} \mathrm{Cl}_{2}\right)$ to give compound $24(0.35 \mathrm{~g}, 48 \%)$ as a clear oil. ${ }^{1} \mathrm{H}$ NMR (400 MHz, $\left.\mathrm{CDCl}_{3}\right): \delta 6.98(\mathrm{~s}, 2 \mathrm{H}, \mathrm{ArH}), 4.38\left(\mathrm{~s}, 2 \mathrm{H}, \mathrm{CH}_{2}\right), 4.37$ (s, $\left.2 \mathrm{H}, \mathrm{CH}_{2}\right), 4.30$ (q, 2 $\left.\mathrm{H}, \mathrm{J}=7.1 \mathrm{~Hz}, \mathrm{OCH}_{2} \mathrm{CH}_{3}\right) 3.47\left(\mathrm{t}, 2 \mathrm{H}, \mathrm{J}=6.6 \mathrm{~Hz}, \mathrm{OCH}_{2} \mathrm{CH}_{2} \mathrm{CH}_{2} \mathrm{CH}_{3}\right), 2.29\left(\mathrm{~s}, 6 \mathrm{H}, \mathrm{Ar}-\mathrm{CH}_{3}\right)$, 1.64-1.55 (m, $\left.2 \mathrm{H}, \mathrm{OCH}_{2} \mathrm{CH}_{2} \mathrm{CH}_{2} \mathrm{CH}_{3}\right), 1.45-1.35\left(\mathrm{~m}, 2 \mathrm{H}, \mathrm{OCH}_{2} \mathrm{CH}_{2} \mathrm{CH}_{2} \mathrm{CH}_{3}\right), 1.33(\mathrm{t}, 3 \mathrm{H}, \mathrm{J}=$ $\left.7.1 \mathrm{~Hz}, \mathrm{OCH}_{2} \mathrm{CH}_{3}\right), 0.92\left(\mathrm{t}, 3 \mathrm{H}, \mathrm{J}=7.5 \mathrm{~Hz}, \mathrm{OCH}_{2} \mathrm{CH}_{2} \mathrm{CH}_{2} \mathrm{CH}_{3}\right) ;{ }^{13} \mathrm{C} \mathrm{NMR}\left(100 \mathrm{MHz}, \mathrm{CDCl}_{3}\right)$ : $\delta 169.1,154.7,134.5,130.6,128.5,72.6,70.4,69.1,61.2,31.8,19.4,16.3,14.2,13.9$; HRMS (FAB) $\mathrm{M}^{+}$calcd for $\mathrm{C}_{17} \mathrm{H}_{26} \mathrm{O}_{4} 294.1831$, found 294.1823.

(4-butoxymethyl-2,6-dimethylphenoxy)-acetic acid (25). A solution of compound 24 (0.35 g, $1.19 \mathrm{mmol})$ and $45 \mathrm{wt} \%$ aqueous $\mathrm{KOH}(7 \mathrm{~mL}, 81.8 \mathrm{mmol})$ in $\mathrm{MeOH}(21 \mathrm{~mL})$ and $\mathrm{THF}(21 \mathrm{~mL})$ was stirred at room temperature for $2 \mathrm{~h}$. The solvent was evaporated under reduced pressure and the remaining solid was taken into $\mathrm{H}_{2} \mathrm{O}(70 \mathrm{~mL})$. The aqueous solution was acidified to $\mathrm{pH} 1$ with $6 \mathrm{~N} \mathrm{HCl}$, extracted with $\mathrm{CH}_{2} \mathrm{Cl}_{2}(2 \times 50 \mathrm{~mL})$, and the combined organic layers were dried over $\mathrm{Na}_{2} \mathrm{SO}_{4}$ and the solvent evaporated under reduced pressure to give compound $25(0.318 \mathrm{~g}$, $100 \%$ ) as a brownish solid. ${ }^{1} \mathrm{H}$ NMR $\left(400 \mathrm{MHz} \mathrm{CDCl}_{3}\right): \delta 7.00$ (s, $\left.2 \mathrm{H}, \mathrm{ArH}\right), 4.41(\mathrm{~s}, 2 \mathrm{H}$, $\left.\mathrm{CH}_{2}\right), 4.38\left(\mathrm{~s}, 2 \mathrm{H}, \mathrm{CH}_{2}\right), 3.46\left(\mathrm{t}, 2 \mathrm{H}, \mathrm{J}=6.5 \mathrm{~Hz}, \mathrm{OCH}_{2} \mathrm{CH}_{2} \mathrm{CH}_{2} \mathrm{CH}_{3}\right), 2.27\left(\mathrm{~s}, 6 \mathrm{H}, \mathrm{Ar}-\mathrm{CH}_{3}\right)$, 1.65-1.54 (m, $\left.2 \mathrm{H}, \mathrm{OCH}_{2} \mathrm{CH}_{2} \mathrm{CH}_{2} \mathrm{CH}_{3}\right), 1.45-1.34\left(\mathrm{~m}, 2 \mathrm{H}, \mathrm{OCH}_{2} \mathrm{CH}_{2} \mathrm{CH}_{2} \mathrm{CH}_{3}\right), 0.92(\mathrm{t}, 3 \mathrm{H}, \mathrm{J}=$ $\left.7.3 \mathrm{~Hz}, \mathrm{OCH}_{2} \mathrm{CH}_{2} \mathrm{CH}_{2} \mathrm{CH}_{3}\right) ;{ }^{13} \mathrm{C} \mathrm{NMR}\left(100 \mathrm{MHz} \mathrm{CDCl}_{3}\right): \delta 153.8,135.0,130.4,128.5,77.2$, 72.4, 70.4, 68.6, 31.8, 19.3, 16.2, 13.9; HRMS (FAB) $\mathrm{M}^{+}$calcd for $\mathrm{C}_{15} \mathrm{H}_{22} \mathrm{O}_{4} 266.1518$, found 266.1516.

\section{G 4 [5'-(2-(4-butoxymethyl-2,6-dimethylphenoxy)-ethoxycarbonylamino)-2',3'-O-di-tert-} butyldimethylsilylguanosine]. A solution of compound $25(0.318 \mathrm{~g}, 1.193 \mathrm{mmol})$ and $\mathrm{SOCl}_{2}$ $(0.85 \mathrm{~mL})$ in benzene $(15 \mathrm{~mL})$ was stirred at reflux under a nitrogen atmosphere for $2 \mathrm{~h}$. The 
solvent evaporated under reduced pressure and the remaining brownish solid was coevaporated with benzene $(2 \times 20 \mathrm{~mL})$ to give (4-butoxymethyl-2,6-dimethylphenoxy)-acetyl chloride 26 ( $0.372 \mathrm{~g}$, IR showed no acid $\mathrm{O}-\mathrm{H}$ stretch and a single $\mathrm{C}=\mathrm{O}$ stretch at $\left.1816 \mathrm{~cm}^{-1}\right)$. A suspension of compound 26 (0.372 g, $1.31 \mathrm{mmol})$, guanosine $15(0.80 \mathrm{~g}, 1.57 \mathrm{mmol})$ and $\mathrm{Et}_{3} \mathrm{~N}(0.91 \mathrm{~mL}, 6.53$ $\mathrm{mmol})$ in $\mathrm{CH}_{2} \mathrm{Cl}_{2}(20 \mathrm{~mL})$ was stirred at room temperature under a nitrogen atmosphere for $24 \mathrm{~h}$. The solvent was evaporated under reduced pressure and the remaining solid was purified by flash chromatography to afford G $4(0.65 \mathrm{~g}, 66 \%)$ as a white solid. ${ }^{1} \mathrm{H}$ NMR (400 MHz, DMSO-d 6 ): $\delta 10.67$ (s, $1 \mathrm{H}, \mathrm{N} 1 \mathrm{H}), 8.51$ (t, $1 \mathrm{H}, \mathrm{J}=6.0 \mathrm{~Hz}$, amide NH), 8.05 (s, $1 \mathrm{H}, \mathrm{H} 8), 6.96$ (s, $2 \mathrm{H}, \operatorname{ArH})$, 6.45 (bs, 2 H, NH$)_{2}$, 5.72 (d, 1 H, J = 7.8 Hz, H1'), 4.79 (dd, 1 H, J = 7.8, 4.3 Hz, H2'), 4.33 (d, 1 $\left.\mathrm{H}, \mathrm{J}=4.3 \mathrm{~Hz}, \mathrm{H}^{\prime}\right), 4.31$ (s, 2H, $\left.\mathrm{CH}_{2}\right) .4 .18$ (s, 2H, $\mathrm{CH}_{2}$ ), 3.99 (dd, $\left.1 \mathrm{H}, \mathrm{J}=8.0,6.0 \mathrm{~Hz}, \mathrm{H} 4{ }^{\prime}\right)$, 3.69-3.56 (m, 1 H, H5'), 3.49-3.39 (m, 1 H, H5"), 3.38 (t, 2H, J = 6.5 Hz, $\mathrm{OCH}_{2} \mathrm{CH}_{2} \mathrm{CH}_{2} \mathrm{CH}_{3}$ ), $2.20\left(\mathrm{~s}, 6 \mathrm{H}, \mathrm{Ar}-\mathrm{CH}_{3}\right), 1.54-1.44\left(\mathrm{~m}, 2 \mathrm{H}, \mathrm{OCH}_{2} \mathrm{CH}_{2} \mathrm{CH}_{2} \mathrm{CH}_{3}\right), 1.37-1.26(\mathrm{~m}, 2 \mathrm{H}$, $\mathrm{OCH}_{2} \mathrm{CH}_{2} \mathrm{CH}_{2} \mathrm{CH}_{3}$ ), 0.90 (s, $9 \mathrm{H}, t$-Bu-Si), 0.86 (t, $3 \mathrm{H}, \mathrm{J}=7.3 \mathrm{~Hz}, \mathrm{OCH}_{2} \mathrm{CH}_{2} \mathrm{CH}_{2} \mathrm{CH}_{3}$ ), 0.71 (s, $9 \mathrm{H}, t$-Bu-Si), 0.11 (s, $\left.3 \mathrm{H}, \mathrm{CH}_{3}-\mathrm{Si}\right), 0.10$ (s, $3 \mathrm{H}, \mathrm{CH}_{3}-\mathrm{Si}$ ), -0.08 (s, $3 \mathrm{H}, \mathrm{CH}_{3}-\mathrm{Si}$ ), -0.34 (s, $3 \mathrm{H}$, $\left.\mathrm{CH}_{3}-\mathrm{Si}\right) ;{ }^{13} \mathrm{C}$ NMR (100 MHz, DMSO-d6): d 168.3, 156.6, 153.6, 153.5, 151.5, 136.3, 134.5, $130.1,130.0,128.1,116.8,85.6,84.4,73.8,73.5,71.5,70.4,69.3,31.3,25.7,25.5,18.9,17.8$, 17.5, 15.8, 13.8, -4.7, -4.8, -5.7; HRMS (FAB) $[\mathrm{M}+\mathrm{H}]^{+}$calcd for $\mathrm{C}_{37} \mathrm{H}_{63} \mathrm{~N}_{6} \mathrm{O}_{7} \mathrm{Si}_{2} 759.4297$, found 759.4312. Anal. calcd for $\mathrm{C}_{37} \mathrm{H}_{62} \mathrm{~N}_{6} \mathrm{O}_{7} \mathrm{Si}_{2}$ : C, 58.54; H, 8.23, found: $\mathrm{C}$, 58.35; H, 8.48. 


\section{Figures.}

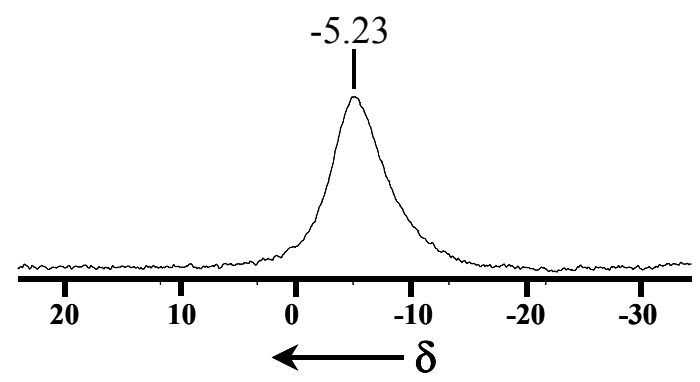

Figure S1. ${ }^{23} \mathrm{Na} \mathrm{NMR} \mathrm{of}(\mathrm{cG} \mathrm{2})_{2} \cdot \mathrm{NaCl} \bullet\left(\mathrm{H}_{2} \mathrm{O}\right)_{\mathrm{n}}$ in water-saturated $\mathrm{CDCl}_{3}(20 \mathrm{mM}$ in cG 2). The spectrum was recorded on a Bruker DRX-500 operating at 132.29 MHz and is reported in ppm relative to $25 \mathrm{mM} \mathrm{NaCl}$ in $\mathrm{D}_{2} \mathrm{O}$ at 0 ppm (external standard).

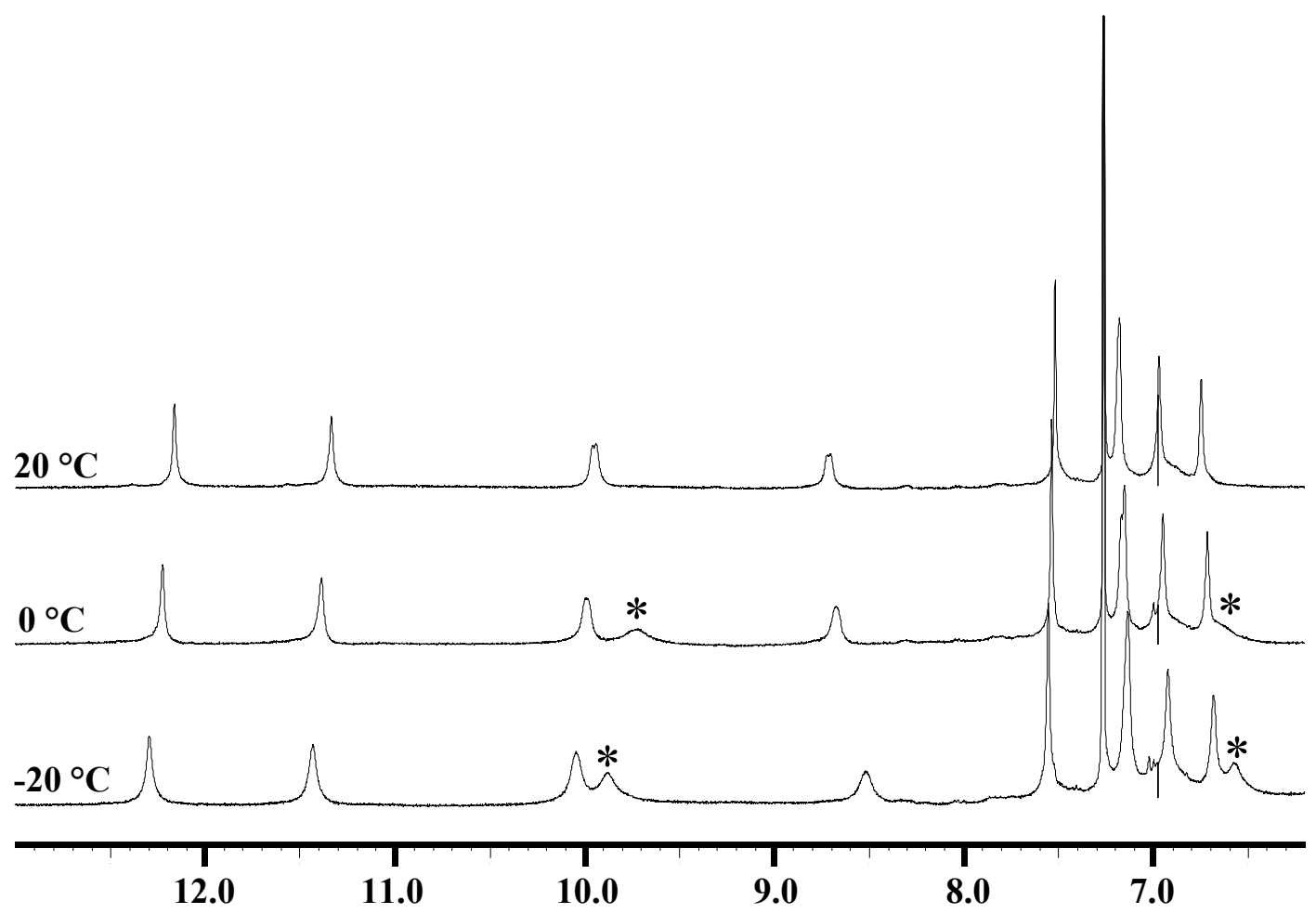

Figure S2. ${ }^{1} \mathrm{H}$ NMR spectra of $(\mathrm{cG} \mathrm{2})_{2} \cdot \mathrm{NaCl} \cdot\left(\mathrm{H}_{2} \mathrm{O}\right)_{\mathrm{n}}$ in water-saturated $\mathrm{CDCl}_{3}$ at $20{ }^{\circ} \mathrm{C}, 0{ }^{\circ} \mathrm{C}$, and $-20{ }^{\circ} \mathrm{C}$. The hydrogen bonded and "free" $\mathrm{N} 2-\mathrm{H}_{\mathrm{i}}$ signals grow in at low temperature and are indicated with asterisks. 


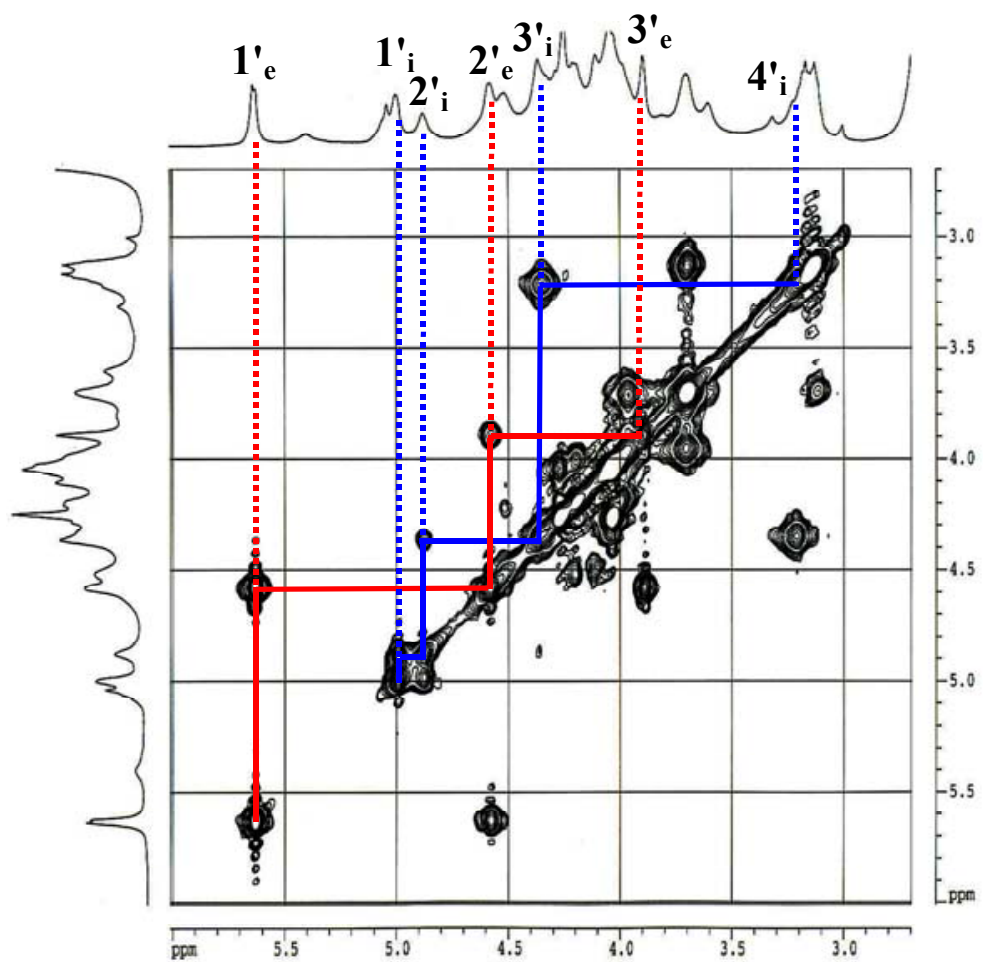

Figure S3. ${ }^{1} \mathrm{H}-{ }^{1} \mathrm{H}$ COSY spectrum of $(\mathrm{cG} \mathrm{2})_{2} \cdot \mathrm{NaCl} \cdot\left(\mathrm{H}_{2} \mathrm{O}\right)_{\mathrm{n}}$ in water-saturated $\mathrm{CDCl}_{3}$ at $-20{ }^{\circ} \mathrm{C}$. Red lines indicate external (e) signal correlations and blue lines indicate internal (i) signal correlations. 


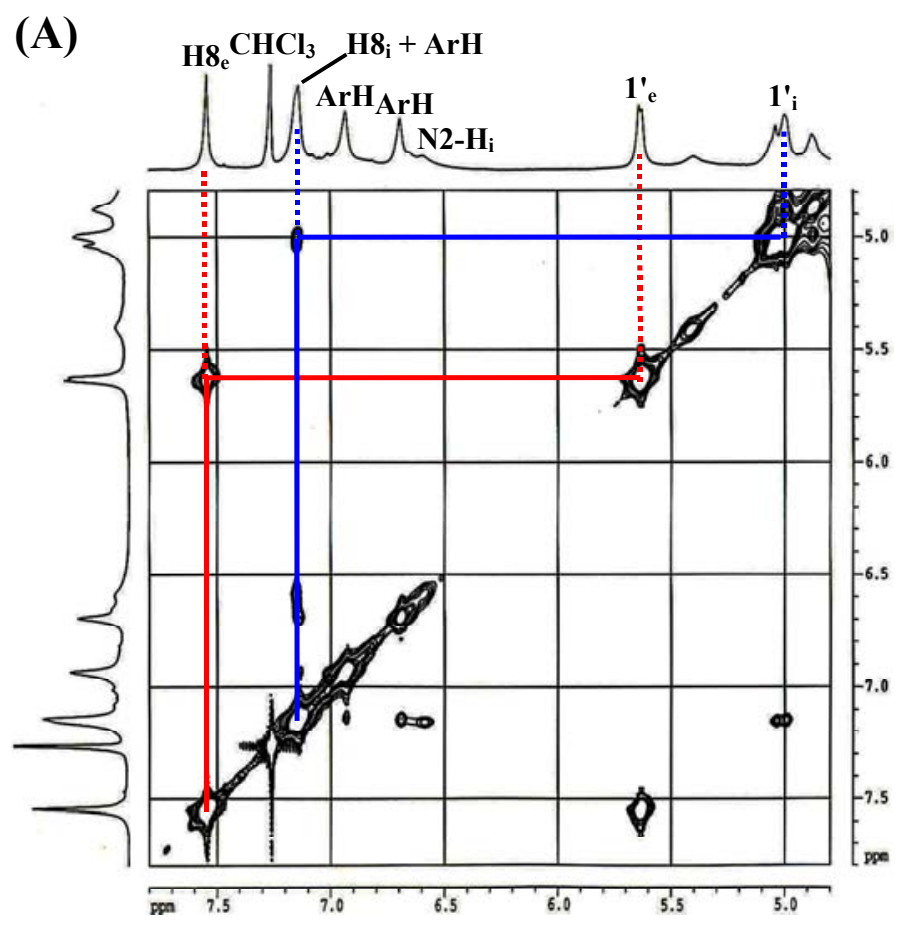

(B)
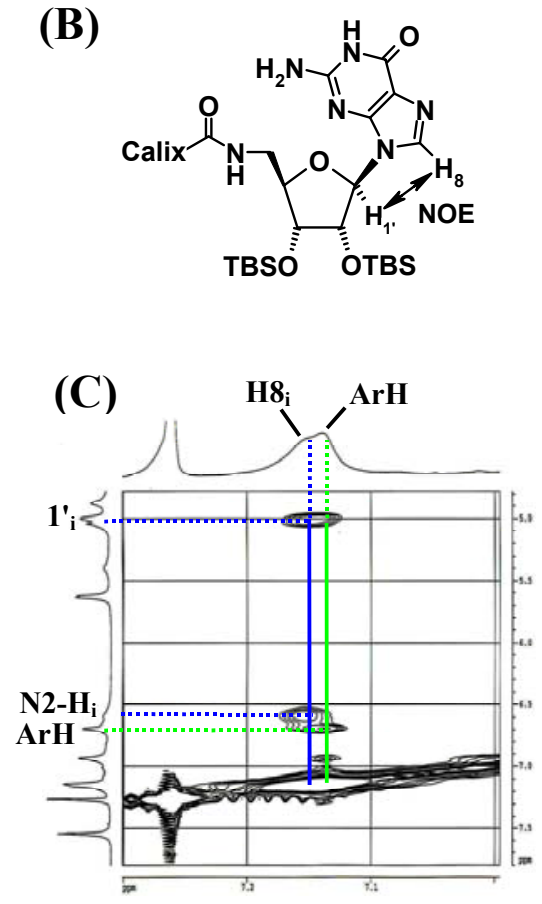

Figure S4. (A) ${ }^{1} \mathrm{H}-{ }^{1} \mathrm{H}$ NOESY spectrum of $(\mathrm{cG} \mathrm{2})_{2} \cdot \mathrm{NaCl} \cdot\left(\mathrm{H}_{2} \mathrm{O}\right)_{\mathrm{n}}$ in water-saturated $\mathrm{CDCl}_{3}$ at -20 ${ }^{\circ} \mathrm{C}$. Red lines indicate external $\mathrm{H} 1^{1}{ }_{\mathrm{e}} \cdots \mathrm{H} 8_{\mathrm{e}}$ signal correlations and blue lines indicate internal $\mathrm{H} 1^{1}{ }_{\mathrm{i}} \cdots \mathrm{H} 8_{\mathrm{i}}$ signal correlations. (B) Guanosine structure indicating the $\mathrm{H} 1^{1} \cdots \mathrm{H} 8$ NOE. (C) Expansion of the F1 (vertical) axis correlations near $\delta 7.15$. Blue lines indicate $H 1^{\prime}{ }_{\mathrm{i}} \cdots \mathrm{H} 8_{\mathrm{i}}$ and N2$\mathrm{H}_{\mathrm{i}} \cdots \mathrm{H} 8_{\mathrm{i}}$ NOEs and green lines indicate ArH $\cdots$ ArH NOEs. The expansion shows that both $\mathrm{H} 8_{\mathrm{i}}$ and an $\mathrm{ArH}$ are indeed present under the signal near $\delta$ 7.15. 


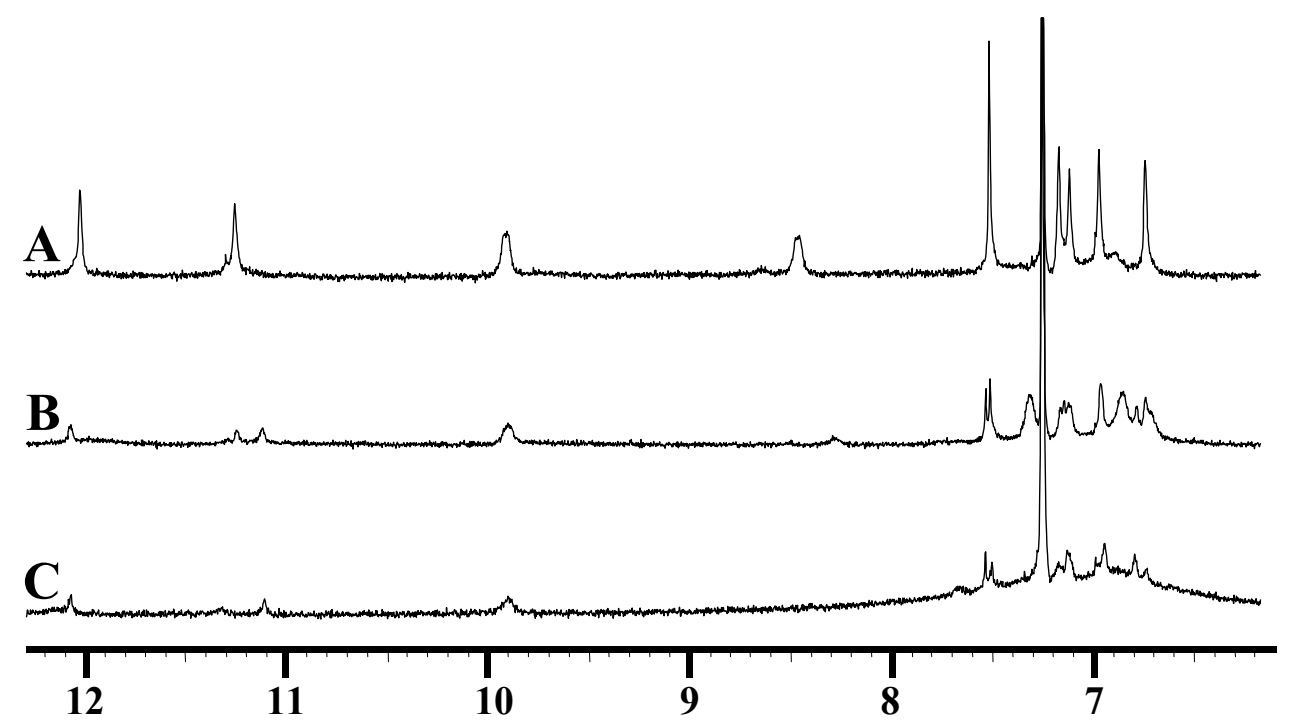

Figure S5. Titration of $1.0 \mathrm{~mL}$ of $(\mathrm{cG} \mathrm{2})_{2} \cdot\left(\mathrm{H}_{2} \mathrm{O}\right)_{\mathrm{n}}$ in water-saturated $\mathrm{CDCl}_{3}(6.7 \mathrm{mM}$ in cG 2) with $0.15 \mathrm{M} \mathrm{NaBPh}_{4}$ in $1: 1 \mathrm{CDCl}_{3}: \mathrm{CD}_{3} \mathrm{CN}$ as followed by NMR: (A) 0 equiv. (B) 0.5 equiv. (22.5 $\mu \mathrm{L})$, and (C) 1.0 equiv. $(45 \mu \mathrm{L})$. 


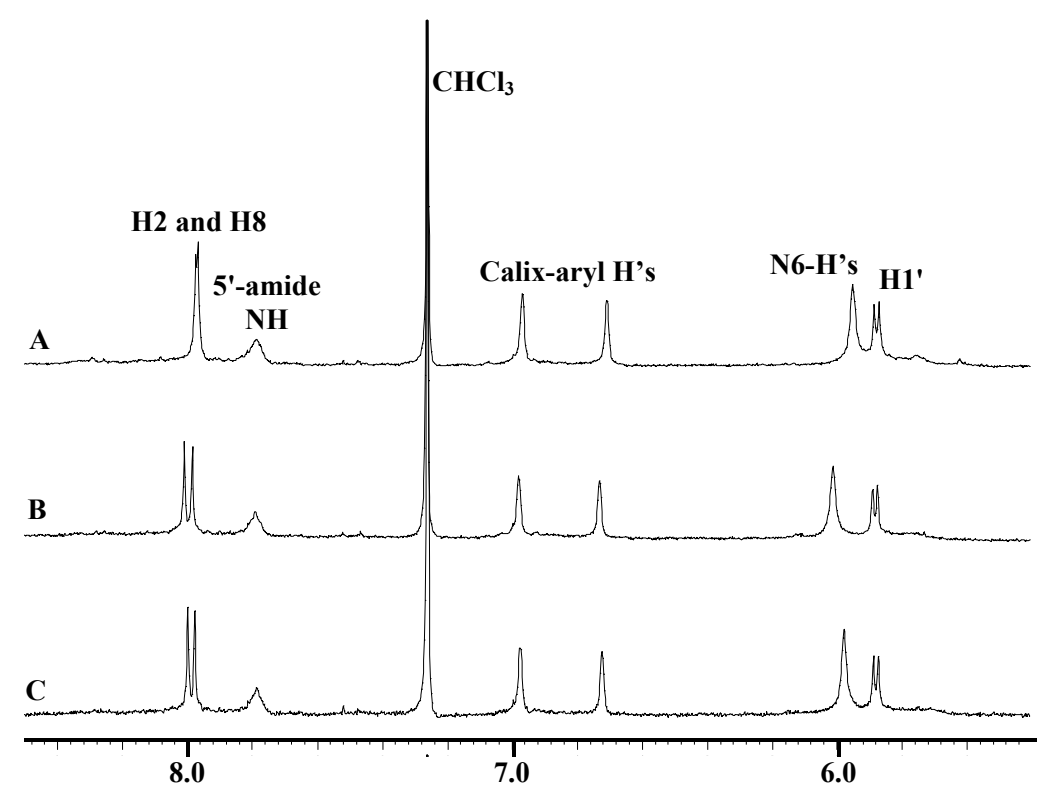

Figure S6. Control experiments with cA 3. ${ }^{1} \mathrm{H}$ NMR spectra of: (A) cA 3 in commercial $\mathrm{CDCl}_{3}$. (B) cA 3 in $\mathrm{CDCl}_{3}$ after washing with $\mathrm{H}_{2} \mathrm{O}$. (C) cA 3 in $\mathrm{CDCl}_{3}$ after washing with $1 \mathrm{M} \mathrm{NaCl}_{(\mathrm{aq})}$. All samples were $2 \mathrm{mM}$ in cA $\mathbf{3}$.

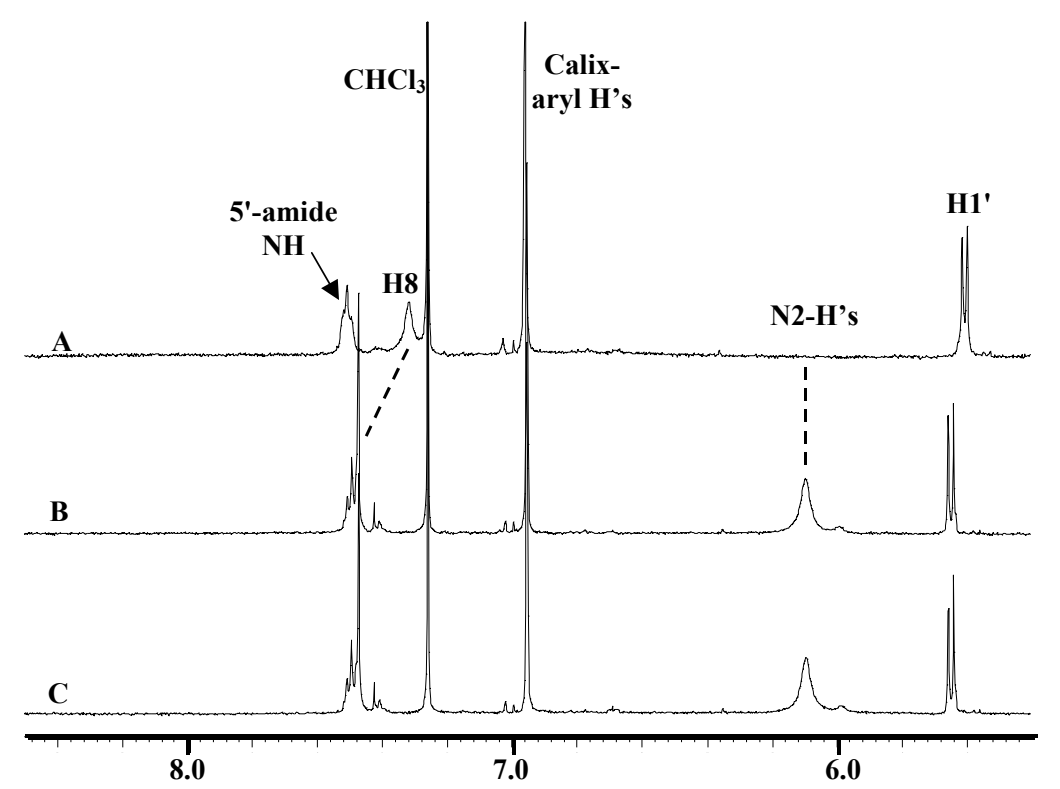

Figure S7. Control experiments with G 4. ${ }^{1} \mathrm{H}$ NMR spectra of: (A) $\mathrm{G} 4$ in commercial $\mathrm{CDCl}_{3}$, (B) $\mathrm{G} 4$ in $\mathrm{CDCl}_{3}$ after washing with $\mathrm{H}_{2} \mathrm{O}$, (C) $\mathrm{G} 4$ in $\mathrm{CDCl}_{3}$ after washing with $1 \mathrm{M} \mathrm{NaCl}_{(\mathrm{aq})}$. All samples were $2 \mathrm{mM}$ in G 4. The changes from A to B \& C indicated by arrows are the shifting of $\mathrm{H} 8(\delta$ 7.3-7.5) and the appearance of $\mathrm{N} 2 \mathrm{H}(\delta 6.1)$. The downfield shift and sharpening of the $\mathrm{H} 8$ resonance of $\mathrm{G} 4$ in water-saturated $\mathrm{CDCl}_{3}$ versus commercial $\mathrm{CDCl}_{3}$ may be due to a change in conformation resulting from hydration of the nucleobase. 


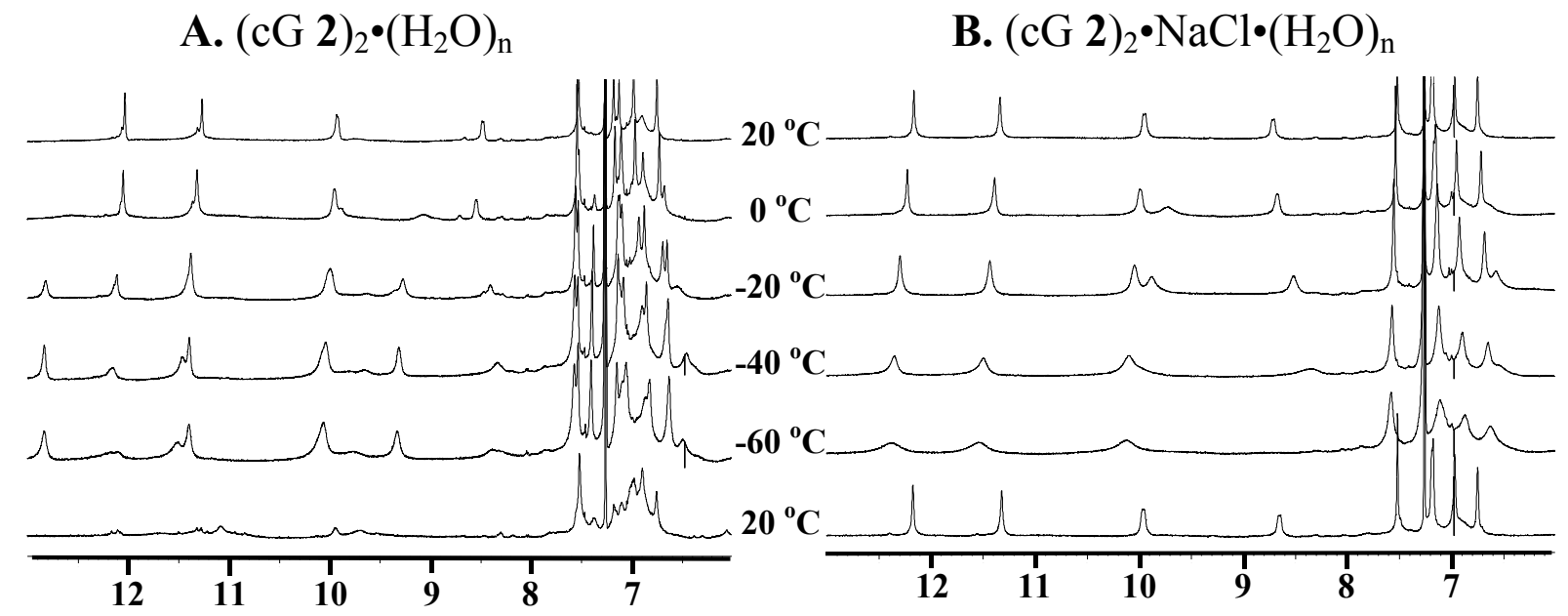

Figure S8. ${ }^{1} \mathrm{H}$ NMR spectra of (A) $(\mathrm{cG} \mathrm{2})_{2} \bullet\left(\mathrm{H}_{2} \mathrm{O}\right)_{\mathrm{n}}$ and (B) $(\mathrm{cG} \mathrm{2})_{2} \bullet \mathrm{NaCl}^{\circ}\left(\mathrm{H}_{2} \mathrm{O}\right)_{\mathrm{n}}$ from $20{ }^{\circ} \mathrm{C}$ down to $-60{ }^{\circ} \mathrm{C}$, then back to $20^{\circ} \mathrm{C}$. The temperature cycle results in decomposition of (cG 2) ${ }_{2} \bullet\left(\mathrm{H}_{2} \mathrm{O}\right)_{n}$ to nonspecific aggregates (bottom spectrum in A) but has no effect on (cG 2) $)_{2} \cdot \mathrm{NaCl} \bullet\left(\mathrm{H}_{2} \mathrm{O}\right)_{\mathrm{n}}$ (bottom spectrum in B). Samples were $2 \mathrm{mM}$ in cG 2. 